\title{
Comparison Between Passive and Active Control of a Non-Linear Dynamical System
}

\author{
S. A. El-Serafi* ${ }^{*}$ M. H. Eissa* ${ }^{*}$ H. M. El-Sherbiny** \\ and T. H. El-GhareEB** \\ * Department of Engineering Mathematics, \\ Faculty of Electronic Engineering, \\ Minufiya University, Menouf 32952, Egypt \\ ** Department of Mathematics, Faculty of Education, \\ Suez Canal University, Suez, Egypt
}

Received March 3, 2005

Revised January 5, 2006

\begin{abstract}
Vibrations and dynamic chaos should be controlled in structures and machines. The wing of the airplane should be free from vibrations or it should be kept minimum. To do so, two main strategies are used. They are passive and active control methods. In this paper we present a mathematical study of passive and active control in some non-linear differential equations describing the vibration of the wing. Firstly, non-linear differential equation representing the wing system subjected to multi-excitation force is considered and solved using the method of multiple scale perturbation. Secondly, a tuned mass absorber (TMA) is applied to the system at simultaneous primary resonance. Thirdly, the same system is considered with 1:2 internal resonance active control absorber. The approximate solution is derived up to the fourth order approximation, the stability of the system is investigated applying both frequency response equations and phase plane methods. Previous work regarding the wing vibration dealt only with a linear system describing its vibration. Some recommendations are given by the end of the work.
\end{abstract}

Key words: control, passive, active, absorber, absorber effectiveness

\section{Introduction}

Vibrations and dynamic chaos are undesired phenomenon in structures. They cause disturbance, discomfort, damage and destruction of the system or the structure. One of the most effective tools for passive vibration control is the dynamic absorber or the damper or the neutralizer [1]. Eissa [2] has shown that the nonlinear absorber widens its range of applications, and its damping coefficient should be kept minimum for better performance [3]. Cheng-Tang Lee et al. [4] demonstrated that a dynamic vibration absorber system can be used to reduce speed fluctuations in rotating machinery. Eissa and El-Ganaini $[5,6]$ studied the control of vibration and dynamic chaos of mechanical structures having quadratic and cubic non-linearities, subjected to harmonic excitation using single and multi-absorbers. Active constrained layer damping (ACLD) has been successfully utilized as effective means of damping out the vibration of various flexible structures [7-12]. In weakly non-linear systems, internal resonances may occur if the linear natural frequencies are commensurate or nearly commensurate, and internal resonances provide coupling and energy exchange among the vibration modes [13, 14]. If two natural 
frequencies of a system with quadratic non-linearities are in the ratio 1:2 there exists a saturation phenomenon [13]. When the system is excited at a frequency near the higher natural frequency, the structure responds at the excitation frequency and the amplitude of the response increases linearly with the excitation amplitude. However, when the high-frequency modal amplitude reaches a critical value, this mode saturates and all additional energy added to the system by increasing the excitation amplitude overflows into the low-frequency mode. Recently the use of internal resonance and saturation phenomena in non-linear control has been extensively studied $[15,18]$. This method is based on an approach originally introduced by Golnaraghi [15] and thoroughly investigated by Nayfeh et al. [17]. To control transient vibrations, reference [15] used a second-order controller coupled to a vibrating system via quadratic or cubic terms. References $[16,17]$ used the saturation phenomenon to successfully control the motion of a d.c. motor with a rigid beam attached.

Aerospace structures are required to be light in weight and hence composite structures are increasingly used. Moreover, aerospace composite structures (e.g. helicopter rotor blades) are often designed with built in elastic bending torsion couplings to improve aerodynamic efficiency. To actively control such structures without too much added weight, PZT (lead zirconate titanate) sensors and actuators are attractive because of their mechanical simplicity, small volume, light weight, large useful bandwidth, efficient conversion of electrical to mechanical energy, ability to perform shape control, and ability to be easily integrated with the structure [19]. Wen, et al. [20] investigated non-linear saturation control, non-linear internal resonance control and linear position-feedback control of steady-state and transient vibrations of a cantilever beam using PZT patches as sensors and actuators.

The aim of this work is to apply both linear passive, and active non-linear vibration absorbers using higher-order internal resonances and saturation phenomena to suppress the steady-state vibrations of a cantilever skew aluminum plate representing the wing of an aircraft [21], [22]. Higher-order internal resonances are introduced using quadratic terms to couple the controller with the plate. The multiple time scale perturbation technique is applied throughout. An approximate solution is derived up to third order approximation. The stability of the system is investigated applying both frequency response functions (FRFs) and phase-plane methods. The effects of the absorber on system behavior are studied numerically. Optimum working conditions of the system are obtained applying both passive and active control methods. Both control methods are demonstrated and compared numerically.

\section{Mathematical Modeling}

The considered system is shown schematically in Fig. 1.

The investigated equation is the modified non-linear differential equation de- 


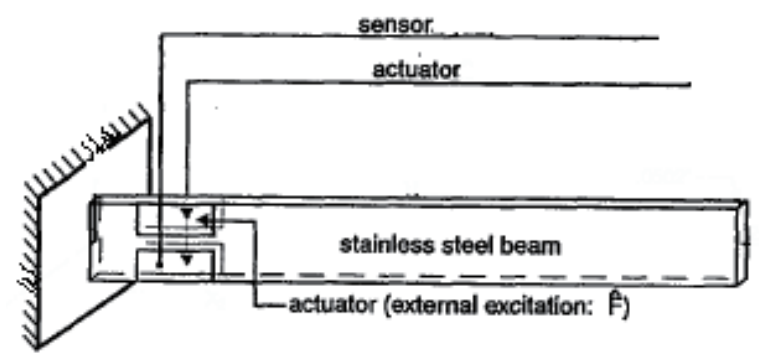

Fig. 1. The non-linear cantilevered skew aluminium plate with a 1:2 non-linear vibration absorber for controlling the first-mode vibration.

scribing the vibration of an aircraft wing [22] which is given by:

$$
\ddot{u}+2 \bar{\zeta} \omega \dot{u}+\omega^{2} u+\bar{\alpha} u^{2}+\bar{\beta} u^{3}=\sum_{j=1}^{N} \bar{F}_{j} \cos \Omega_{j} t
$$

where $u, \dot{u}$ and $\ddot{u}$ represent displacement, velocity and acceleration of the vibrating system, $\omega$ is the natural frequency, $\bar{\zeta}$ is the damping coefficient, $\bar{\alpha}$ and $\bar{\beta}$ are nonlinear stiffness coefficients, $\bar{F}_{j}$ is the forcing amplitudes and $\Omega_{j}$ are the excitation frequencies.

\subsection{Perturbation analysis}

We seek a first-order approximate solution of equation (1) by using the method of multiple scales [2] in the form

$$
u\left(T_{0}, T_{n} ; \varepsilon\right)=\sum_{k=0}^{\infty} \varepsilon^{k} u_{k}\left(T_{0}, T_{n}\right)
$$

where $\varepsilon$ is a small dimensionless parameter used for book-keeping only; $T_{0}=t$ and $T_{n}=\varepsilon^{n} t$ where $n=(1,2, \ldots)$ are the fast and slow time scales respectively. To make dampings, non-linearities, and the primary resonance force appear in the same perturbation equations, we order that $\bar{\zeta}=\varepsilon \zeta, \bar{\alpha}=\varepsilon \alpha, \bar{\beta}=\varepsilon \beta, \bar{F}_{j}=\varepsilon F_{j}$. Substituting equation (2) into equation (1) and equating coefficients of like powers of $\varepsilon$, we obtain the following set of ordinary differential equations:

$$
\begin{aligned}
\left(D_{0}^{2}+\omega^{2}\right) u_{0}= & 0 \\
\left(D_{0}^{2}+\omega^{2}\right) u_{1}= & -2 D_{0} D_{1} u_{0}-2 \zeta \omega D_{0} u_{0}-\alpha u_{0}^{2}-\beta u_{0}^{3}+\sum_{j=1}^{N} F_{j} \cos \Omega_{j} T_{0} \\
\left(D_{0}^{2}+\omega^{2}\right) u_{2}= & -D_{1}^{2} u_{0}-2 D_{0} D_{1} u_{1}-2 \zeta \omega\left(D_{1} u_{0}+D_{0} u_{1}\right)-2 \alpha u_{0} u_{1}-3 \beta u_{0}^{2} u_{1} \\
\left(D_{0}^{2}+\omega^{2}\right) u_{3}= & -D_{1}^{2} u_{1}-2 D_{0} D_{1} u_{2}-2 \zeta \omega\left(D_{1} u_{1}+D_{0} u_{2}\right)-\alpha\left(u_{1}^{2}+2 u_{0} u_{2}\right) \\
& -3 \beta\left(u_{1}^{2} u_{0}+u_{0}^{2} u_{2}\right)
\end{aligned}
$$


where

$$
\frac{d}{d t}=D_{0}+\varepsilon D_{1}+\varepsilon^{2} D_{2}+\cdots, \quad D_{i}=\frac{\partial}{\partial T_{i}}, i=0,1,2, \ldots .
$$

For our considered case we have the external excitation frequency will be $\Omega_{j}$, $j=1,2,3,4$ for simplicity.

The solution of equation (3) can be expressed in the form

$$
u_{0}\left(T_{0}, T_{n}\right)=A_{0}\left(T_{n}\right) e^{i \omega T_{0}}+c c
$$

where $A_{0}$ is a complex function in $T_{n}$ and $c c$ represents the complex conjugate. Substituting equation (7) into equation (4), yields

$$
\begin{aligned}
\left(D_{0}^{2}+\omega^{2}\right) u_{1}=[ & \left.-2 i \omega\left(D_{1} A_{0}+\zeta \omega A_{0}\right)-3 \beta A_{0}^{2} \bar{A}_{0}\right] e^{i \omega T_{0}}-\alpha A_{0}^{2} e^{2 i \omega T_{0}} \\
& -2 \alpha A_{0} \bar{A}_{0}-\beta A_{0}^{3} e^{3 i \omega T_{0}}+\frac{1}{2} \sum_{j=1}^{4} F_{j} e^{i \Omega_{j} T_{0}}+c c
\end{aligned}
$$

Eliminating the secular terms, then the first order approximation is given by

$$
u_{1}\left(T_{0}, T_{n}\right)=E_{1} e^{i \omega T_{0}}+E_{2} e^{2 i \omega T_{0}}+E_{3} e^{3 i \omega T_{0}}+E_{4}+\sum_{j=1}^{4} E_{(4+j)} e^{i \Omega_{j} T_{0}}+c c
$$

where $E_{m}(m=1,2,3,4)$ are complex functions in $T_{n}$. From equation (7) and (9) into equation (5), yields

$$
\begin{aligned}
\left(D_{0}^{2}+\right. & \left.\omega^{2}\right) u_{2}=\left[-D_{1}^{2} A_{0}-2 i \omega D_{1} A_{1}-2 \zeta \omega D_{1} A_{0}-2 i \zeta \omega^{2} A_{1}+\frac{10 \alpha^{2}}{3 \omega^{2}} A_{0}^{2} \bar{A}_{0}\right. \\
& \left.-\frac{3 \beta^{2}}{8 \omega^{2}} A_{0}^{3} \bar{A}_{0}^{2} 6 \beta A_{0} \bar{A}_{0} A_{1}-3 \beta \bar{A}_{1} A_{0}^{2}\right] e^{i \omega T_{0}} \\
& +\left[-\frac{8 i \alpha}{3 \omega} A_{0} D_{1} A_{0}-\frac{4 i \alpha}{3} \zeta A_{0}^{2}+\frac{15 \alpha \beta}{4 \omega^{2}} A_{0}^{3} \bar{A}_{0}-2 \alpha A_{0} A_{1}\right] e^{2 i \omega T_{0}} \\
& -\left[\frac{9 i \beta}{4 \omega} A_{0}^{2} D_{1} A_{0}+\frac{3 i \beta}{4} \zeta A_{0}^{3}+\frac{2 \alpha^{2}}{3 \omega^{2}} A_{0}^{3}+\frac{3 \beta^{2}}{4 \omega^{2}} A_{0}^{4} \bar{A}_{0}+3 \beta A_{0}^{2} A_{1}\right] e^{3 i \omega T_{0}} \\
& -\sum_{j=1}^{4} \frac{F_{j}}{\omega^{2}-\Omega_{j}^{2}}\left[\left(i \zeta \omega \Omega_{j}+3 \beta A_{0} \bar{A}_{0}\right) e^{i \Omega_{j} T_{0}}+\alpha A_{0}\left(e^{i\left(\omega+\Omega_{j}\right) T_{0}}+e^{i\left(\omega-\Omega_{j}\right) T_{0}}\right)\right. \\
& \left.+\frac{3}{2} \beta A_{0}^{2}\left(e^{i\left(2 \omega+\Omega_{j}\right) T_{0}}+e^{i\left(2 \omega-\Omega_{j}\right) T_{0}}\right)\right]-\frac{5 \alpha \beta}{4 \omega^{2}} A_{0}^{4} e^{4 i \omega T_{0}}-\frac{3 \beta^{2}}{8 \omega^{2}} A_{0}^{5} e^{5 i \omega T_{0}} \\
& +\frac{10 \alpha \beta}{\omega^{2}} A_{0}^{2} \bar{A}_{0}^{2}+A_{0} \bar{A}_{1}+A_{1} \bar{A}_{0}+c c
\end{aligned}
$$

To obtain a bounded solution the secular terms should be eliminated, then the second order approximation is given by

$$
u_{2}\left(T_{0}, T_{n}\right)=H_{1} e^{i \omega T_{0}}+H_{2} e^{2 i \omega T_{0}}+H_{3} e^{3 i \omega T_{0}}+H_{4} e^{4 i \omega T_{0}}+H_{5} e^{5 i \omega T_{0}}
$$




$$
\begin{aligned}
& +\sum_{j=1}^{4}\left[H_{(5+j)} e^{i \Omega_{j} T_{0}}+H_{(9+j)} e^{i\left(\omega+\Omega_{j}\right) T_{0}}+H_{(13+j)} e^{i\left(\omega-\Omega_{j}\right) T_{0}}\right. \\
& \left.+H_{(17+j)} e^{i\left(2 \omega+\Omega_{j}\right) T_{0}}+H_{(21+j)} e^{i\left(2 \omega-\Omega_{j}\right) T_{0}}\right]+H_{26}+c c
\end{aligned}
$$

where $H_{m}(m=1,2, \ldots, 26)$ are complex functions in $T_{n}$. From (7), (9) and (11) into (6), we get the third order approximation as:

$$
\begin{aligned}
& u_{3}\left(T_{0}, T_{n}\right)=P_{1} e^{i \omega T_{0}}+P_{2} e^{2 i \omega T_{0}}+P_{3} e^{3 i \omega T_{0}}+P_{4} e^{4 i \omega T_{0}}+P_{5} e^{5 i \omega T_{0}}+P_{6} e^{6 i \omega T_{0}} \\
& +P_{7} e^{7 i \omega T_{0}}+P_{8} e^{i \Omega_{1} T_{0}}+P_{9} e^{i \Omega_{2} T_{0}}+P_{10} e^{i \Omega_{3} T_{0}}+P_{11} e^{i \Omega_{4} T_{0}} \\
& +P_{12} e^{2 i \Omega_{1} T_{0}}+P_{13} e^{2 i \Omega_{2} T_{0}}+P_{14} e^{2 i \Omega_{3} T_{0}}+P_{15} e^{2 i \Omega_{4} T_{0}}+P_{16} e^{i\left(2 \Omega_{1}+\omega\right) T_{0}} \\
& +P_{17} e^{i\left(2 \Omega_{2}+\omega\right) T_{0}}+P_{18} e^{i\left(2 \Omega_{3}+\omega\right) T_{0}}+P_{19} e^{i\left(2 \Omega_{4}+\omega\right) T_{0}}+P_{20} e^{i\left(2 \Omega_{1}-\omega\right) T_{0}} \\
& +P_{21} e^{i\left(2 \Omega_{2}-\omega\right) T_{0}}+P_{22} e^{i\left(2 \Omega_{3}-\omega\right) T_{0}}+P_{23} e^{i\left(2 \Omega_{4}-\omega\right) T_{0}}+P_{24} e^{i\left(\Omega_{1}+\omega\right) T_{0}} \\
& +P_{25} e^{i\left(\Omega_{2}+\omega\right) T_{0}}+P_{26} e^{i\left(\Omega_{3}+\omega\right) T_{0}}+P_{27} e^{i\left(\Omega_{4}+\omega\right) T_{0}}+P_{28} e^{i\left(\Omega_{1}-\omega\right) T_{0}} \\
& +P_{29} e^{i\left(\Omega_{2}-\omega\right) T_{0}}+P_{30} e^{i\left(\Omega_{3}-\omega\right) T_{0}}+P_{31} e^{i\left(\Omega_{4}-\omega\right) T_{0}}+P_{32} e^{i\left(\Omega_{1}+2 \omega\right) T_{0}} \\
& +P_{33} e^{i\left(\Omega_{2}+2 \omega\right) T_{0}}+P_{34} e^{i\left(\Omega_{3}+2 \omega\right) T_{0}}+P_{35} e^{i\left(\Omega_{4}+2 \omega\right) T_{0}}+P_{36} e^{i\left(\Omega_{1}-2 \omega\right) T_{0}} \\
& +P_{37} e^{i\left(\Omega_{2}-2 \omega\right) T_{0}}+P_{38} e^{i\left(\Omega_{3}-2 \omega\right) T_{0}}+P_{39} e^{i\left(\Omega_{4}-2 \omega\right) T_{0}}+P_{40} e^{i\left(\Omega_{1}+3 \omega\right) T_{0}} \\
& +P_{41} e^{i\left(\Omega_{2}+3 \omega\right) T_{0}}+P_{42} e^{i\left(\Omega_{3}+3 \omega\right) T_{0}}+P_{43} e^{i\left(\Omega_{4}+3 \omega\right) T_{0}}+P_{44} e^{i\left(\Omega_{1}-3 \omega\right) T_{0}} \\
& +P_{45} e^{i\left(\Omega_{2}-3 \omega\right) T_{0}}+P_{46} e^{i\left(\Omega_{3}-3 \omega\right) T_{0}}+P_{47} e^{i\left(\Omega_{4}-3 \omega\right) T_{0}}+P_{48} e^{i\left(\Omega_{1}+4 \omega\right) T_{0}} \\
& +P_{49} e^{i\left(\Omega_{2}+4 \omega\right) T_{0}}+P_{50} e^{i\left(\Omega_{3}+4 \omega\right) T_{0}}+P_{51} e^{i\left(\Omega_{4}+4 \omega\right) T_{0}}+P_{52} e^{i\left(\Omega_{1}-4 \omega\right) T_{0}} \\
& +P_{53} e^{i\left(\Omega_{2}-4 \omega\right) T_{0}}+P_{54} e^{i\left(\Omega_{3}-4 \omega\right) T_{0}}+P_{55} e^{i\left(\Omega_{4}-4 \omega\right) T_{0}} \\
& +P_{56}\left[e^{i\left(\Omega_{2}+\Omega_{1}\right) T_{0}}+e^{i\left(\Omega_{2}-\Omega_{1}\right) T_{0}}\right]+P_{57}\left[e^{i\left(\Omega_{3}+\Omega_{1}\right) T_{0}}+e^{i\left(\Omega_{3}-\Omega_{1}\right) T_{0}}\right] \\
& +P_{58}\left[e^{i\left(\Omega_{3}+\Omega_{2}\right) T_{0}}+e^{i\left(\Omega_{3}-\Omega_{2}\right) T_{0}}\right]+P_{59}\left[e^{i\left(\Omega_{4}+\Omega_{1}\right) T_{0}}+e^{i\left(\Omega_{4}-\Omega_{1}\right) T_{0}}\right] \\
& +P_{60}\left[e^{i\left(\Omega_{4}+\Omega_{2}\right) T_{0}}+e^{i\left(\Omega_{4}-\Omega_{2}\right) T_{0}}\right]+P_{61}\left[e^{i\left(\Omega_{4}+\Omega_{3}\right) T_{0}}+e^{i\left(\Omega_{4}-\Omega_{3}\right) T_{0}}\right] \\
& +P_{62} e^{i\left[\left(\Omega_{2}+\Omega_{1}\right)+\omega\right] T_{0}}+P_{63} e^{i\left[\left(\Omega_{2}+\Omega_{1}\right)-\omega\right] T_{0}}+P_{64} e^{i\left[\left(\Omega_{2}-\Omega_{1}\right)+\omega\right] T_{0}} \\
& +P_{65} e^{i\left[\left(\Omega_{2}-\Omega_{1}\right)-\omega\right] T_{0}}+P_{66} e^{i\left[\left(\Omega_{3}+\Omega_{1}\right)+\omega\right] T_{0}}+P_{67} e^{i\left[\left(\Omega_{3}+\Omega_{1}\right)-\omega\right] T_{0}} \\
& +P_{68} e^{i\left[\left(\Omega_{3}-\Omega_{1}\right)+\omega\right] T_{0}}+P_{69} e^{i\left[\left(\Omega_{3}-\Omega_{1}\right)-\omega\right] T_{0}}+P_{70} e^{i\left[\left(\Omega_{3}+\Omega_{2}\right)+\omega\right] T_{0}} \\
& +P_{71} e^{i\left[\left(\Omega_{3}+\Omega_{2}\right)-\omega\right] T_{0}}+P_{72} e^{i\left[\left(\Omega_{3}-\Omega_{2}\right)+\omega\right] T_{0}}+P_{73} e^{i\left[\left(\Omega_{3}-\Omega_{2}\right)-\omega\right] T_{0}} \\
& +P_{74} e^{i\left[\left(\Omega_{4}+\Omega_{1}\right)+\omega\right] T_{0}}+P_{75} e^{i\left[\left(\Omega_{4}+\Omega_{1}\right)-\omega\right] T_{0}}+P_{76} e^{i\left[\left(\Omega_{4}-\Omega_{1}\right)+\omega\right] T_{0}} \\
& +P_{77} e^{i\left[\left(\Omega_{4}-\Omega_{1}\right)-\omega\right] T_{0}}+P_{78} e^{i\left[\left(\Omega_{4}+\Omega_{2}\right)+\omega\right] T_{0}}+P_{79} e^{i\left[\left(\Omega_{4}+\Omega_{2}\right)-\omega\right] T_{0}} \\
& +P_{80} e^{i\left[\left(\Omega_{4}-\Omega_{2}\right)+\omega\right] T_{0}}+P_{81} e^{i\left[\left(\Omega_{4}-\Omega_{2}\right)-\omega\right] T_{0}}+P_{82} e^{i\left[\left(\Omega_{4}+\Omega_{3}\right)+\omega\right] T_{0}} \\
& +P_{83} e^{i\left[\left(\Omega_{4}+\Omega_{3}\right)-\omega\right] T_{0}}+P_{84} e^{i\left[\left(\Omega_{4}-\Omega_{3}\right)+\omega\right] T_{0}}+P_{85} e^{i\left[\left(\Omega_{4}-\Omega_{3}\right)-\omega\right] T_{0}} \\
& +P_{86}+c c
\end{aligned}
$$

where $P_{m}(m=1,2, \ldots, 55,62,63, \ldots, 86)$ are complex functions in $T_{n}$. From the above analysis the solution of $u$ is given by

$$
u=u_{0}+\varepsilon u_{1}+\varepsilon^{2} u_{2}+\varepsilon^{3} u_{3}+O\left(\varepsilon^{4}\right)
$$

From the above-derived solutions, the reported resonance cases are deduced as shown in Table 1. 
Table 1.

\begin{tabular}{|c|l|l|}
\hline No. & \multicolumn{1}{|c|}{ Type of Resonances } & Case \\
\hline 1 & trivial resonance & $\Omega_{j} \cong \omega=0$ \\
\hline 2 & primary resonance & $\Omega_{j} \cong \omega$ \\
\hline 3 & sub-harmonic resonances & $\Omega_{j} \cong n \omega, \quad n=2,3,4$ \\
\hline 4 & super-harmonic resonances & $\Omega_{j} \cong \omega / 2$ \\
\hline 5 & combined resonances & $\pm \omega \cong \pm \Omega_{1} \pm \Omega_{2}, \pm \omega \cong \pm \Omega_{1} \pm \Omega_{3}$, \\
& & $\pm \omega \cong \pm \Omega_{1} \pm \Omega_{4}, \pm \omega \cong \pm \Omega_{2} \pm \Omega_{3}$, \\
& & $\pm \omega \cong \pm \Omega_{2} \pm \Omega_{4}, \pm \omega \cong \pm \Omega_{3} \pm \Omega_{4}$ \\
& & $\pm \omega \cong \frac{1}{2}\left( \pm \Omega_{1} \pm \Omega_{2}\right), \pm \omega \cong \frac{1}{2}\left( \pm \Omega_{1} \pm \Omega_{3}\right)$ \\
& & $\pm \omega \cong \frac{1}{2}\left( \pm \Omega_{1} \pm \Omega_{4}\right), \pm \omega \cong \frac{1}{2}\left( \pm \Omega_{2} \pm \Omega_{3}\right)$ \\
& & $\pm \omega \cong \frac{1}{2}\left( \pm \Omega_{2} \pm \Omega_{4}\right), \pm \omega \cong \frac{1}{2}\left( \pm \Omega_{3} \pm \Omega_{4}\right)$ \\
\hline 6 & simultaneous resonance & any combination of the two resonance cases \\
& & are classified as simultaneous resonance \\
\hline
\end{tabular}

\subsection{Stability of the system}

After studying numerically the different resonance cases, one of the worst cases has been chosen to study the system stability. The selected resonance case is the primary resonance one where $\Omega_{j} \cong \omega$. In this case we introduce a detuning parameter $\sigma$ such that

$$
\Omega_{j}=\omega+\varepsilon \sigma, \quad j=1,2,3,4
$$

Eliminating the secular terms of the first order approximation given by equation (8) leads to the solvability conditions for the first order approximation. Using equation (14) and noting that $A_{0}$ is a function in $T_{1}$ only, we get

$$
-2 i \omega D_{1} A_{0}-2 i \zeta \omega^{2} A_{0}-3 \beta A_{0}^{2} \bar{A}_{0}+\frac{1}{2} F_{j} e^{i \sigma T_{1}}=0
$$

Substituting the polar form $A_{0}=\frac{1}{2} a\left(T_{1}\right) e^{i \gamma\left(T_{1}\right)}$ into equation (15), we get

$$
-2 i \omega\left(\frac{1}{2} a^{\prime}+\frac{1}{2} i a \gamma^{\prime}\right) e^{i \gamma}-2 i \zeta \omega^{2}\left(\frac{1}{2} a e^{i \gamma}\right)-3 \beta\left(\frac{1}{8} a^{3} e^{i \gamma}\right)+\frac{1}{2} F_{j} e^{i \sigma T_{1}}=0
$$

which yields:

$$
a^{\prime}+i a \gamma^{\prime}+\zeta \omega a-\frac{3 i \beta a^{3}}{8 \omega}+\frac{i F_{j}}{2 \omega}(\cos \mu+i \sin \mu)=0
$$

where $\mu=\sigma T_{1}-\gamma, \mu^{\prime}=\sigma-\gamma^{\prime}$, separating real and imaginary parts in equation (17), we get

$$
a^{\prime}+\zeta \omega a-\frac{F_{j}}{2 \omega} \sin \mu=0 \quad a\left(\sigma-\mu^{\prime}\right)-\frac{3 \beta a^{3}}{8 \omega}+\frac{F_{j}}{2 \omega} \cos \mu=0
$$


Then for steady state solution $a^{\prime}=\mu^{\prime}=0$, and equation (18) becomes

$$
\zeta \omega=\frac{F_{j}}{2 a \omega} \sin \mu \quad \sigma-\frac{3 \beta a^{2}}{8 \omega}=-\frac{F_{j}}{2 a \omega} \cos \mu
$$

squaring equation (19), and adding the results, we get

$$
\sigma^{2}-\frac{3 \beta a^{2}}{4 \omega} \sigma+\left(\zeta^{2} \omega^{2}+\frac{9 \beta^{2} a^{4}}{64 \omega^{2}}-\frac{F_{j}^{2}}{4 a^{2} \omega^{2}}\right)=0 \Rightarrow \sigma=\frac{3 \beta a^{2}}{8 \omega} \pm \sqrt{F_{j}^{2}-4 a^{2} \zeta^{2} \omega^{4}}
$$

\section{Stability of the fixed points}

To analyze the stability of the fixed points, one lets

$$
a=b_{0}+b_{1}\left(T_{1}\right), \quad \mu=\mu_{0}+\mu_{1}\left(T_{1}\right)
$$

where $b_{0}$ and $\mu_{0}$ satisfy equation (18) and $b_{1}$ and $\mu_{1}$ are perturbation terms which are assumed to be small compared to $b_{0}$ and $\mu_{0}$, substituting equation (21) into equation (18), the following are obtained

$$
\begin{aligned}
& \dot{b}_{1}+\Gamma_{1} b_{1}+\Gamma_{2} \mu_{1}=0 \\
& \hat{\mu}_{1}+\Gamma_{1} \mu_{1}+\Gamma_{3} b_{1}=0
\end{aligned}
$$

where

$$
\Gamma_{1}=\zeta \omega, \quad \Gamma_{2}=b_{0}\left(\sigma-\frac{3 \beta b_{0}^{2}}{8 \omega}\right) \quad \text { and } \quad \Gamma_{3}=\frac{1}{b_{0}}\left(\mu_{0}+\frac{9 \beta b_{0}^{2}}{8 \omega}-\sigma\right)
$$

The eigenvalues of the above system of equations (22) and (23) can be obtained as follows

$$
\left|\begin{array}{cc}
\left(\lambda+\Gamma_{1}\right) & \Gamma_{2} \\
\Gamma_{3} & \left(\lambda+\Gamma_{1}\right)
\end{array}\right|=0
$$

i.e.

$$
\lambda^{2}+2 \lambda \Gamma_{1}+\left(\Gamma_{1}^{2}-\Gamma_{2} \Gamma_{3}\right)=0 \text { or } \lambda=-\Gamma_{1} \pm \sqrt{\Gamma_{2} \Gamma_{3}}
$$

Hence, the non-linear solution is stable if and only if the real part of $\lambda \prec 0$, i.e. $\Gamma_{1}^{2} \succ \Gamma_{2} \Gamma_{3}$ otherwise it is unstable.

\subsection{Results and discussion}

Results are presented in graphical forms as steady state amplitude against detuning parameter $(\sigma)$ and as time history. A good criterion of both stability and dynamic chaos presence is the phase-plane trajectories, which are shown for some considered cases. The solutions of the frequency response equations regarding the stability of the system is shown in Fig. 2. It can be seen from the figure that maximum steady state amplitude occurs at primary resonance when $\Omega_{j} \cong \omega$. Figs. $2(\mathrm{a})-(\mathrm{d})$ show the effects of the damping coefficient $\zeta$, the non-linear parameter $\beta$, the natural frequency $\omega$ and the excitation amplitudes $F_{j}$ on the steady 
state amplitude "a". Fig. 2 (a) shows that the curve is bent to the right, leading to the occurrence of the jump phenomena and the effect of increasing or decreasing $\zeta$ is insignificant denoting the occurrence of the saturation phenomena. Fig. 2 (b) shows that the positive and negative values of $\beta$, produce either hard or soft spring respectively as the curve is either bent to the right or the left, leading to the appearance of the jump phenomenon. Fig. 2 (c) indicates that the steady state amplitude is monotonic decreasing function in $\omega$ also for the decreasing natural frequency $\omega$, the curve is bent to the right leading to the occurrence of the jump phenomena. Fig. 2 (d) shows that the steady state amplitude is a monotonic increasing function in the excitation amplitude $F_{j}$.

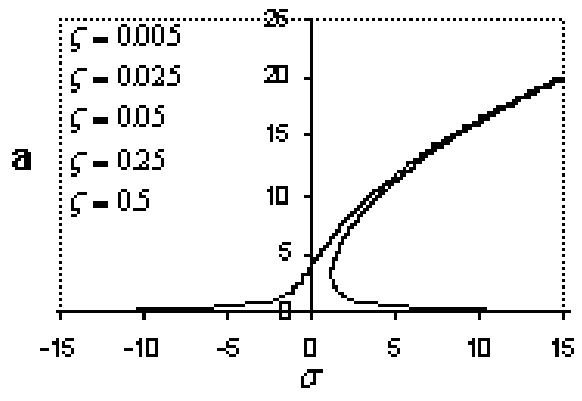

(a) Effects of damping coefficient $\zeta$

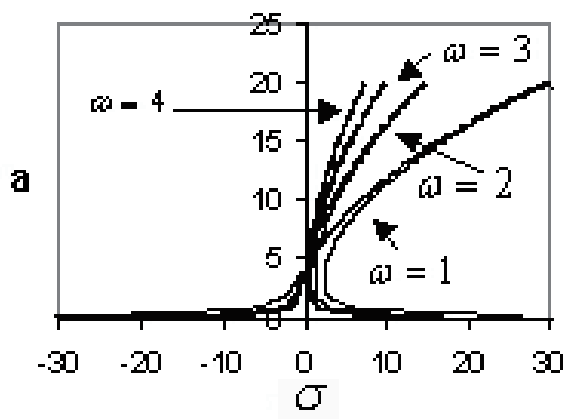

(c) Effects of natural frequency

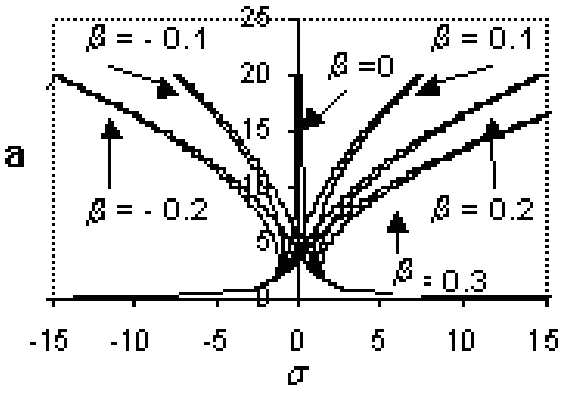

(b) Effects of non-linear parameter $\beta$

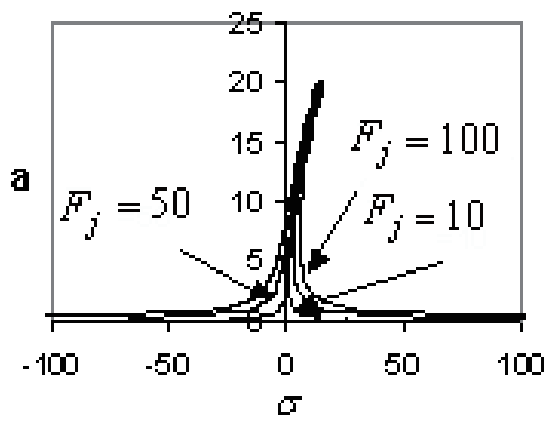

(d) Effects of excitation amplitude $F_{j}$

Fig. 2 .

Fig. 3 illustrates the response and the phase plane for the non-resonant system at some practical values of the equation parameters. It can be seen from the figure that the maximum steady state amplitude is about $30 \%$ of the maximum excitation amplitude $F_{1}$. The phase-plane shows approximately fine limit cycle denoting that the system is free of chaos. 

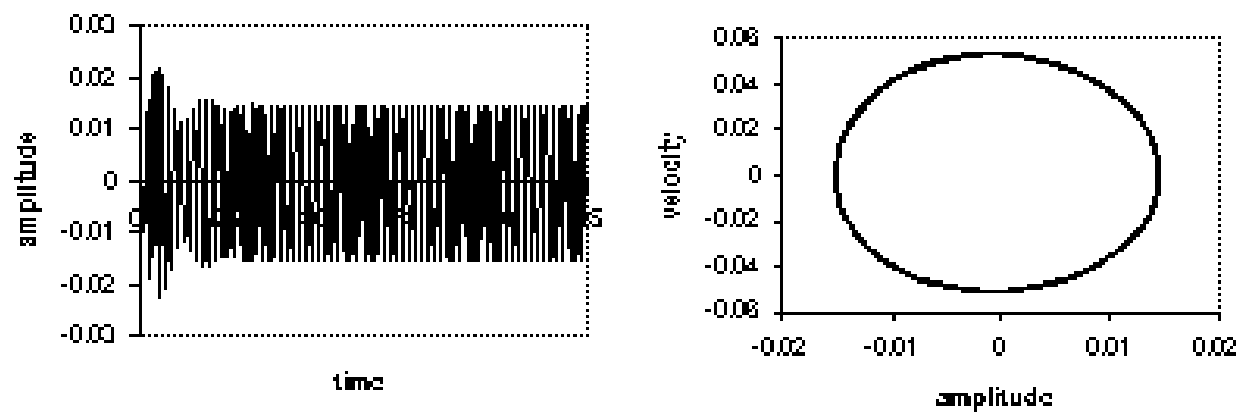

Fig. 3. Non-resonance system behavior (basic case). $\zeta=0.08, \omega=3, \alpha=0.05$, $\beta=0.2, F_{1}=0.05, F_{2}=0.0125, F_{3}=0.0025, F_{4}=0.0005, \Omega_{1}=3.5, \Omega_{2}=7$, $\Omega_{3}=10.5, \Omega_{4}=14$.

\subsection{Resonance cases}

Some of the deduced resonance cases are confirmed numerically (Fig. 4). Table 2 summarizes the different considered cases. It can be seen that the primary resonance case or cases represent the system worst case.
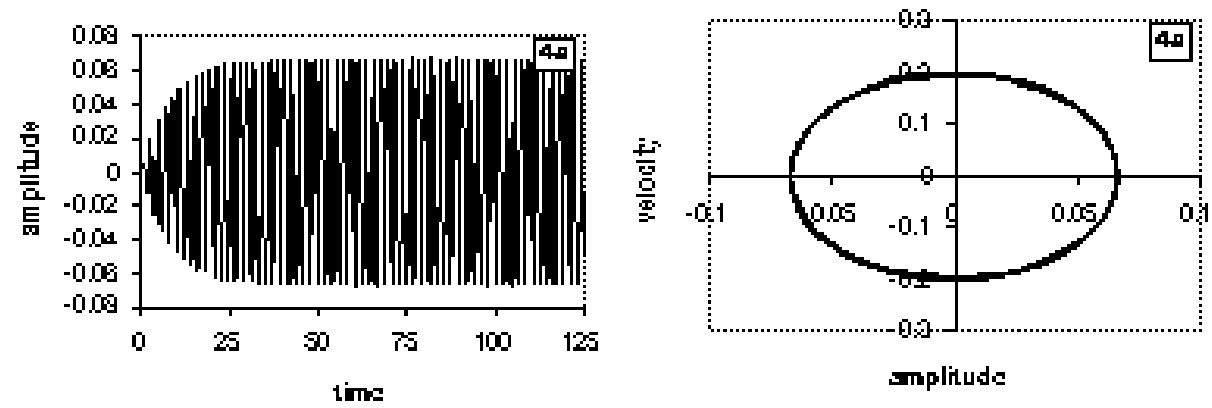

(a) Primary resonance $\Omega_{1} \cong \omega$
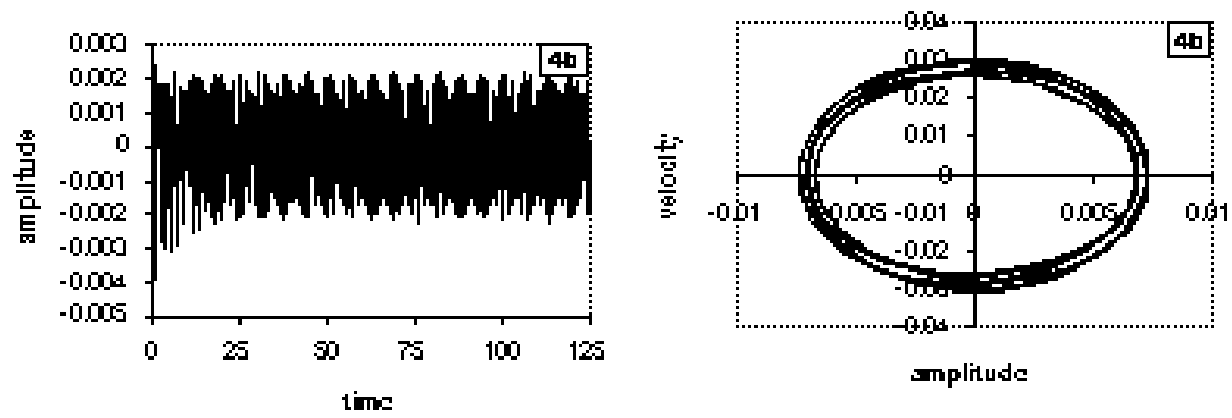

(b) Sub-harmonic resonance $\Omega_{1} \cong 2 \omega$ 

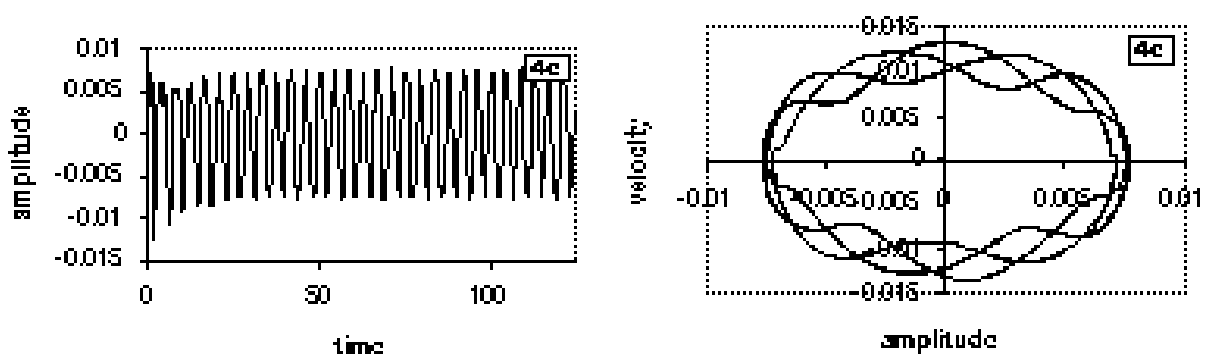

(c) Super-harmonic resonance $\Omega_{1} \cong \omega / 2$
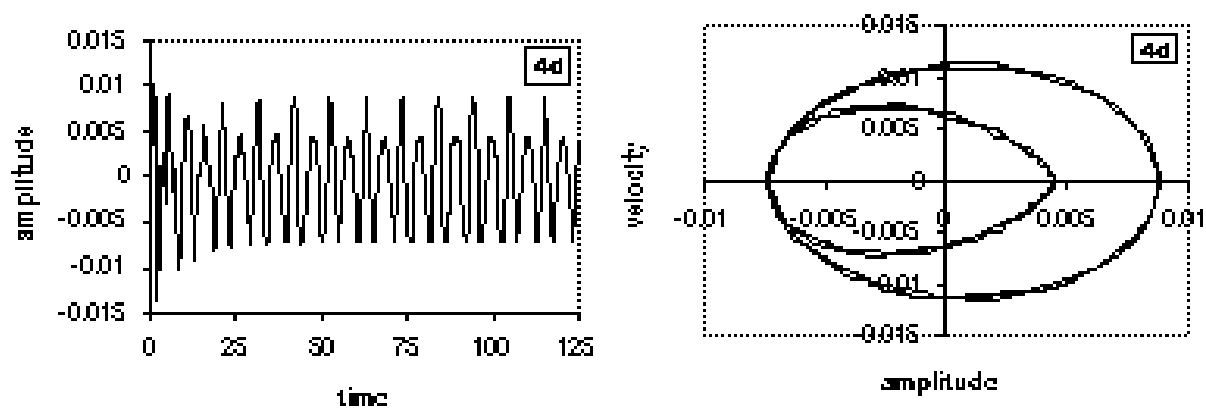

(d) Combined resonance $\omega \cong \Omega_{1}+\Omega_{2}$
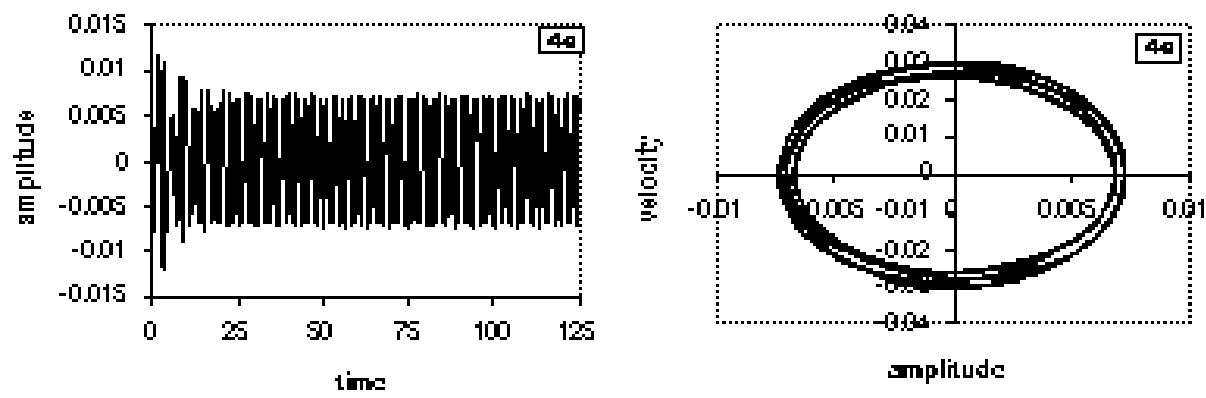

(e) Combined resonance $\omega \cong \Omega_{2}-\Omega_{1}$
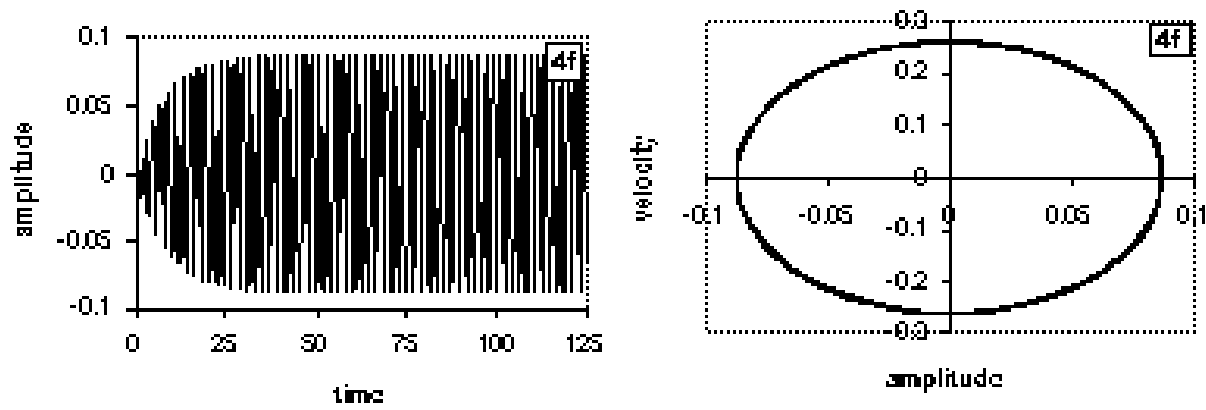

(f) Simultaneous resonance $\Omega_{1} \cong \Omega_{2} \cong \Omega_{3} \cong \Omega_{4} \cong \omega$

Fig. 4. 
Table 2. Summary of some investigated resonance cases.

\begin{tabular}{|c|c|c|c|c|}
\hline No & Resonance type & Case & Amplitude ratio & Chaos \\
\hline 1 & & Non-resonant & $100 \%$ & Limit cycle \\
\hline 2 & \multirow{2}{*}{$\begin{array}{l}\text { Primary } \\
\text { resonance }\end{array}$} & $\Omega_{1} \cong \omega$ & $460 \%$ & Limit cycle \\
\hline 3 & & $\Omega_{2} \cong \omega$ & $215 \%$ & Multi-Limit cycle \\
\hline 4 & \multirow{6}{*}{$\begin{array}{l}\text { Sub-harmonic } \\
\text { resonance }\end{array}$} & $\Omega_{1} \cong 2 \omega$ & $15 \%$ & Multi-Limit cycle \\
\hline 5 & & $\Omega_{2} \cong 2 \omega$ & $105 \%$ & Double-Limit \\
\hline 6 & & $\Omega_{1} \cong 3 \omega$ & $10 \%$ & Multi-Limit cycle \\
\hline 7 & & $\Omega_{2} \cong 3 \omega$ & $105 \%$ & Limit cycle \\
\hline 8 & & $\Omega_{1} \cong 4 \omega$ & $5 \%$ & Multi-Limit cycle \\
\hline 9 & & $\Omega_{2} \cong 4 \omega$ & $100 \%$ & Limit cycle \\
\hline 10 & \multirow{2}{*}{$\begin{array}{l}\text { Super-harmonic } \\
\text { resonance }\end{array}$} & $\Omega_{1} \cong \omega / 2$ & $50 \%$ & Multi-Limit cycle \\
\hline 11 & & $\Omega_{2} \cong \omega / 2$ & $115 \%$ & Multi-Limit cycle \\
\hline 12 & \multirow{4}{*}{$\begin{array}{l}\text { Combined } \\
\text { resonance }\end{array}$} & $\omega \cong \Omega_{1}+\Omega_{2}$ & $60 \%$ & Double-Limit cycle \\
\hline 13 & & $\omega \cong \Omega_{2}-\Omega_{1}$ & $105 \%$ & Limit cycle \\
\hline 14 & & $2 \omega \cong \Omega_{1}+\Omega_{2}$ & $150 \%$ & Multi-Limit cycle \\
\hline 15 & & $2 \omega \cong \Omega_{2}-\Omega_{1}$ & $105 \%$ & Limit cycle \\
\hline 16 & $\begin{array}{l}\text { Simultaneous } \\
\text { resonance }\end{array}$ & $\omega \cong \Omega_{1} \cong \Omega_{2}$ & $570 \%$ & Limit cycle \\
\hline
\end{tabular}

\section{Passive Control}

Using a linear tuned mass absorber (TMA) connected to the system, equations of motions can be written in the following form:

$$
\begin{aligned}
& \ddot{u}_{2}+2 \varepsilon \zeta \omega \dot{u}_{2}+\omega^{2} u_{2}+\varepsilon \alpha u_{2}^{2}+\varepsilon \beta u_{2}^{3}+\varepsilon \zeta_{1}\left(\dot{u}_{2}-\dot{u}_{1}\right)+\varepsilon \gamma\left(u_{2}-u_{1}\right) \\
& \quad=\varepsilon \sum_{j=1}^{N} F_{j} \cos \Omega_{j} t \\
& \ddot{u}_{1}+2 \varepsilon \zeta_{2} \omega_{1}\left(\dot{u}_{1}-\dot{u}_{2}\right)+\omega_{1}^{2}\left(u_{1}-u_{2}\right)=0
\end{aligned}
$$

where $u_{1}$ denotes the response of the second-order controller, $u_{2}$ represents one of the modal co-ordinates of a structure, $\omega$ and $\omega_{1}$ are the natural frequencies, $\zeta$ and $\zeta_{2}$ are the damping coefficients, $\alpha$ and $\beta$ are non-linear coefficients of the wing, $\varepsilon$ is a small perturbation parameter, $F_{j}$ the forcing amplitudes and $\Omega_{j}$ are the excitation frequencies, $j=1,2$ for simplicity. Following the same procedure as in part 1 , we get:

$$
\begin{aligned}
& u_{20}\left(T_{0}, T_{n}\right)=A_{0}\left(T_{n}\right) e^{i \omega T_{0}}+c c \\
& u_{10}\left(T_{0}, T_{n}\right)=B_{0}\left(T_{n}\right) e^{i \omega_{1} T_{0}}+C_{0}\left(T_{n}\right) e^{i \omega T_{0}}+c c
\end{aligned}
$$




$$
\begin{aligned}
& u_{21}\left(T_{0}, T_{n}\right)=K_{1} e^{i \omega T_{0}}+K_{2} e^{2 i \omega T_{0}}+K_{3} e^{3 i \omega T_{0}}+K_{4} e^{i \omega_{1} T_{0}}+K_{5} e^{i \Omega_{1} T_{0}} \\
& +K_{6} e^{i \Omega_{2} T_{0}}+K_{7}+c c \\
& u_{11}\left(T_{0}, T_{n}\right)=K_{8} e^{i \omega_{1} T_{0}}+K_{9} e^{i \omega T_{0}}+K_{10} e^{2 i \omega T_{0}}+K_{11} e^{3 i \omega T_{0}}+K_{12} e^{i \Omega_{1} T_{0}} \\
& +K_{13} e^{i \Omega_{2} T_{0}}+K_{14}+c c \\
& u_{22}\left(T_{0}, T_{n}\right)=R_{1} e^{i \omega T_{0}}+R_{2} e^{2 i \omega T_{0}}+R_{3} e^{3 i \omega T_{0}}+R_{4} e^{4 i \omega T_{0}}+R_{5} e^{5 i \omega T_{0}}+R_{6} e^{i \omega_{1} T_{0}} \\
& +R_{7} e^{i\left(\omega_{1}+\omega\right) T_{0}}+R_{8} e^{i\left(\omega_{1}-\omega\right) T_{0}}+R_{9} e^{i\left(\omega_{1}+2 \omega\right) T_{0}}+R_{10} e^{i\left(\omega_{1}-2 \omega\right) T_{0}} \\
& +R_{11} e^{i \Omega_{1} T_{0}}+R_{12} e^{i \Omega_{2} T_{0}}+R_{13} e^{i\left(\omega+\Omega_{1}\right) T_{0}}+R_{14} e^{i\left(\omega+\Omega_{2}\right) T_{0}} \\
& +R_{15} e^{i\left(\omega-\Omega_{1}\right) T_{0}}+R_{16} e^{i\left(\omega-\Omega_{2}\right) T_{0}}+R_{17} e^{i\left(2 \omega+\Omega_{1}\right) T_{0}}+R_{18} e^{i\left(2 \omega+\Omega_{2}\right) T_{0}} \\
& +R_{19} e^{i\left(2 \omega-\Omega_{1}\right) T_{0}}+R_{20} e^{i\left(2 \omega-\Omega_{2}\right) T_{0}}+R_{21}+c c \\
& u_{12}\left(T_{0}, T_{n}\right)=P_{1} e^{i \omega T_{0}}+P_{2} e^{2 i \omega T_{0}}+P_{3} e^{3 i \omega T_{0}}+P_{4} e^{4 i \omega T_{0}}+P_{5} e^{5 i \omega T_{0}}+P_{6} e^{i \omega_{1} T_{0}} \\
& +P_{7} e^{i\left(\omega_{1}+\omega\right) T_{0}}+P_{8} e^{i\left(\omega_{1}-\omega\right) T_{0}}+P_{9} e^{i\left(\omega_{1}+2 \omega\right) T_{0}}+P_{10} e^{i\left(\omega_{1}-2 \omega\right) T_{0}} \\
& +P_{11} e^{i \Omega_{1} T_{0}}+P_{12} e^{i \Omega_{2} T_{0}}+P_{13} e^{i\left(\omega+\Omega_{1}\right) T_{0}}+P_{14} e^{i\left(\omega+\Omega_{2}\right) T_{0}} \\
& +P_{15} e^{i\left(\omega-\Omega_{1}\right) T_{0}}+P_{16} e^{i\left(\omega-\Omega_{2}\right) T_{0}}+P_{17} e^{i\left(2 \omega+\Omega_{1}\right) T_{0}}+P_{18} e^{i\left(2 \omega+\Omega_{2}\right) T_{0}} \\
& +P_{19} e^{i\left(2 \omega-\Omega_{1}\right) T_{0}}+P_{20} e^{i\left(2 \omega-\Omega_{2}\right) T_{0}}+P_{21}+c c \\
& u_{23}\left(T_{0}, T_{n}\right)=q_{1} e^{i \omega T_{0}}+q_{2} e^{2 i \omega T_{0}}+q_{3} e^{3 i \omega T_{0}}+q_{4} e^{4 i \omega T_{0}}+q_{5} e^{5 i \omega T_{0}}+q_{6} e^{6 i \omega T_{0}} \\
& +q_{7} e^{7 i \omega T_{0}}+q_{8} e^{i \omega_{1} T_{0}}+q_{9} e^{2 i \omega_{1} T_{0}}+q_{10} e^{i\left(\omega_{1}+\omega\right) T_{0}}+q_{11} e^{i\left(\omega_{1}-\omega\right) T_{0}} \\
& +q_{12} e^{i\left(\omega_{1}+2 \omega\right) T_{0}}+q_{13} e^{i\left(\omega_{1}-2 \omega\right) T_{0}}+q_{14} e^{i \Omega_{1} T_{0}}+q_{15} e^{i \Omega_{2} T_{0}} \\
& +q_{16} e^{2 i \Omega_{1} T_{0}}+q_{17} e^{2 i \Omega_{2} T_{0}}+q_{18} e^{i\left(\omega+\Omega_{1}\right) T_{0}}+q_{19} e^{i\left(\omega+\Omega_{2}\right) T_{0}} \\
& +q_{20} e^{i\left(\omega-\Omega_{1}\right) T_{0}}+q_{21} e^{i\left(\omega-\Omega_{2}\right) T_{0}}+q_{22} e^{i\left(2 \omega+\Omega_{1}\right) T_{0}}+q_{23} e^{i\left(2 \omega+\Omega_{2}\right) T_{0}} \\
& +q_{24} e^{i\left(2 \omega-\Omega_{1}\right) T_{0}}+q_{25} e^{i\left(2 \omega-\Omega_{2}\right) T_{0}}+q_{26} e^{i\left(\Omega_{1}+\Omega_{2}\right) T_{0}}+q_{27} e^{i\left(\Omega_{2}-\Omega_{1}\right) T_{0}} \\
& +q_{28} e^{i\left(\omega+\left(\Omega_{1}+\Omega_{2}\right)\right) T_{0}}+q_{29} e^{i\left(\omega+\left(\Omega_{2}-\Omega_{1}\right)\right) T_{0}}+q_{30} e^{i\left(\omega-\left(\Omega_{1}+\Omega_{2}\right)\right) T_{0}} \\
& +q_{31} e^{i\left(\omega-\left(\Omega_{2}-\Omega_{1}\right)\right) T_{0}}+q_{32}+c c \\
& u_{13}\left(T_{0}, T_{n}\right)=g_{1} e^{i \omega T_{0}}+g_{2} e^{2 i \omega T_{0}}+g_{3} e^{3 i \omega T_{0}}+g_{4} e^{4 i \omega T_{0}}+g_{5} e^{5 i \omega T_{0}}+g_{6} e^{6 i \omega T_{0}} \\
& +g_{7} e^{7 i \omega T_{0}}+g_{8} e^{i \omega_{1} T_{0}}+g_{9} e^{2 i \omega_{1} T_{0}}+g_{10} e^{i\left(\omega_{1}+\omega\right) T_{0}}+g_{11} e^{i\left(\omega_{1}-\omega\right) T_{0}} \\
& +g_{12} e^{i\left(\omega_{1}+2 \omega\right) T_{0}}+g_{13} e^{i\left(\omega_{1}-2 \omega\right) T_{0}}+g_{14} e^{i \Omega_{1} T_{0}}+g_{15} e^{i \Omega_{2} T_{0}} \\
& +g_{16} e^{2 i \Omega_{1} T_{0}}+g_{17} e^{2 i \Omega_{2} T_{0}}+g_{18} e^{i\left(\omega+\Omega_{1}\right) T_{0}}+g_{19} e^{i\left(\omega+\Omega_{2}\right) T_{0}} \\
& +g_{20} e^{i\left(\omega-\Omega_{1}\right) T_{0}}+g_{21} e^{i\left(\omega-\Omega_{2}\right) T_{0}}+g_{22} e^{i\left(2 \omega+\Omega_{1}\right) T_{0}}+g_{23} e^{i\left(2 \omega+\Omega_{2}\right) T_{0}} \\
& +g_{24} e^{i\left(2 \omega-\Omega_{1}\right) T_{0}}+g_{25} e^{i\left(2 \omega-\Omega_{2}\right) T_{0}}+g_{26} e^{i\left(\Omega_{1}+\Omega_{2}\right) T_{0}}+g_{27} e^{i\left(\Omega_{2}-\Omega_{1}\right) T_{0}} \\
& +g_{28} e^{i\left(\omega+\left(\Omega_{1}+\Omega_{2}\right)\right) T_{0}}+g_{29} e^{i\left(\omega+\left(\Omega_{2}-\Omega_{1}\right)\right) T_{0}}+g_{30} e^{i\left(\omega-\left(\Omega_{1}+\Omega_{2}\right)\right) T_{0}} \\
& +g_{31} e^{i\left(\omega-\left(\Omega_{2}-\Omega_{1}\right)\right) T_{0}}+g_{32}+c c
\end{aligned}
$$

where $A_{0}, B_{0}, C_{0}, K_{i}, R_{m}, P_{m}, q_{n}, g_{n}(i=1,2, \ldots, 14),(m=1,2, \ldots, 21)$ and $(n=1,2, \ldots, 32)$ are complex functions in $T_{n}$. From the above analysis the general solutions of $u_{2}$ and $u_{1}$ is given by

$$
u_{2}=u_{20}+\varepsilon u_{21}+\varepsilon^{2} u_{22}+\varepsilon^{3} u_{23}+O\left(\varepsilon^{4}\right) \text { and } u_{1}=u_{10}+\varepsilon u_{11}+\varepsilon^{2} u_{12}+\varepsilon^{3} u_{13}+O\left(\varepsilon^{3}\right)
$$
Many resonance cases are shown in Table 3 . 
Table 3 .

\begin{tabular}{|c|l|l|}
\hline No. & \multicolumn{1}{|c|}{ Type of Resonances } & \multicolumn{1}{c|}{ Case } \\
\hline 1 & trivial resonance & $\Omega_{1} \cong \Omega_{2} \cong \omega \cong \omega_{1}=0$ \\
\hline 2 & primary resonance & $\Omega_{1} \cong \omega, \Omega_{2} \cong \omega, \Omega_{1} \cong \omega_{1}, \Omega_{2} \cong \omega_{1}$ \\
\hline 3 & sub-harmonic resonances & $\Omega_{1} \cong n \omega, \Omega_{2} \cong n \omega, \omega_{1}=m \omega$ \\
& & $n=2,3$ and $m=2,3, \ldots, 7$ \\
\hline 4 & super-harmonic resonances & $\Omega_{1} \cong \omega / 2, \Omega_{2} \cong \omega / 2, \Omega_{1} \cong \omega / 3, \Omega_{2} \cong \omega / 3$, \\
& & $\omega_{1}=\omega / 2, \Omega_{1} \cong \omega_{1} / 2, \Omega_{2} \cong \omega_{1} / 2$ \\
\hline 5 & combined resonances & $\pm \omega \cong \pm \Omega_{1} \pm \Omega_{2}, \pm \omega_{1} \cong \pm \Omega_{1} \pm \Omega_{2}$, \\
& & $\pm \omega \cong \frac{1}{2}\left( \pm \Omega_{1} \pm \Omega_{2}\right), \pm \Omega_{1} \cong \pm \omega \pm \omega_{1}$, \\
& & $\pm \Omega_{2} \cong \pm \omega \pm \omega_{1}, \pm \Omega_{1} \cong \pm 2 \omega \pm \omega_{1}$, \\
& & $\pm \Omega_{2} \cong \pm 2 \omega \pm \omega_{1}, \pm \Omega_{1} \pm \Omega_{2}= \pm \omega \pm \omega_{1}$ \\
\hline 6 & simultaneous resonance & any combination of the two resonance cases \\
& & are classified as simultaneous resonance \\
\hline
\end{tabular}

\subsection{Stability of the system}

We study the stability of the system at the simultaneous primary resonance $\Omega_{1} \cong \omega, \Omega_{2} \cong \omega_{1}$. Using the detuning $\sigma_{1}$ and $\sigma_{2}$ such that

$$
\Omega_{1} \cong \omega+\varepsilon \sigma_{1} \text { and } \Omega_{2} \cong \omega_{1}+\varepsilon \sigma_{2}
$$

Eliminating the secular terms of equations (28) and (29), leads to the solvability conditions for the first order approximation and noting that $A_{0}$ and $B_{0}$ are a function in $T_{1}$ only, we get

$$
\begin{aligned}
& {\left[-2 i \omega\left(D_{1} A_{0}+\zeta \omega A_{0}\right)-3 \beta A_{0}^{2} \bar{A}_{0}-i \omega \zeta_{1} A_{0}-\gamma A_{0}+\frac{\omega_{1}^{2} A_{0}}{\omega_{1}^{2}-\omega^{2}}\left(i \omega \zeta_{1}+\gamma\right)\right] e^{i \omega T_{0}}} \\
& \quad+\frac{1}{2} F_{1} e^{i \Omega_{1} T_{0}}=0 \\
& -\left[2 i \omega_{1}\left(D_{1} B_{0}+\zeta_{2} \omega_{1} B_{0}\right)+\frac{\omega_{1}^{2} B_{0}}{\omega_{1}^{2}-\omega^{2}}\left(i \omega_{1} \zeta_{1}+\gamma\right)\right] e^{i \omega_{1} T_{0}} \\
& \quad+\frac{\omega_{1}^{2} F_{2}}{2\left(\omega^{2}-\Omega_{2}^{2}\right)} e^{i \Omega_{2} T_{0}}=0
\end{aligned}
$$

putting

$$
A_{0}=\frac{1}{2} a_{1}\left(T_{1}\right) e^{i m\left(T_{1}\right)} \text { and } B_{0}=\frac{1}{2} a_{2}\left(T_{1}\right) e^{i n\left(T_{1}\right)}
$$

similar to the former system, there are three possibilities in addition to the trivial solution. They are
(1) $a_{1} \neq 0, a_{2}=0$
(2) $a_{2} \neq 0, a_{1}=0$
(3) $a_{1} \neq 0, a_{2} \neq 0$ 
The corresponding frequency response function (FRF) for each case are:

$$
\begin{aligned}
\sigma_{1}^{2} & -\left(\frac{3 \beta a_{1}^{2}}{4 \omega}+\frac{\gamma}{\omega}-\frac{\gamma \omega_{1}^{3}}{\omega^{2}\left(\omega_{1}^{2}-\omega^{2}\right)}\right) \sigma_{1}+\left[\frac{9 \beta^{2} a_{1}^{4}}{64 \omega^{2}}+\frac{\gamma^{2}}{4 \omega^{2}}+\frac{\gamma^{2} \omega_{1}^{4}}{4 \omega^{2}\left(\omega_{1}^{2}-\omega^{2}\right)^{2}}\right. \\
& +\frac{3 \beta \gamma a_{1}^{2}}{4 \omega^{2}}-\frac{3 \beta \gamma \omega_{1}^{2} a_{1}^{2}}{8 \omega^{2}\left(\omega_{1}^{2}-\omega^{2}\right)}-\frac{\gamma^{2} \omega_{1}^{2}}{2 \omega^{2}\left(\omega_{1}^{2}-\omega^{2}\right)}+\zeta^{2} \omega^{2}+\frac{\zeta_{1}^{2}}{4}+\frac{\omega_{1}^{4} \zeta_{1}^{2}}{4\left(\omega_{1}^{2}-\omega^{2}\right)^{2}} \\
& \left.+\zeta \zeta_{1} \omega-\frac{\omega \omega_{1}^{2} \zeta \zeta_{1}}{\omega_{1}^{2}-\omega^{2}}-\frac{\omega_{1}^{2} \zeta_{1}^{2}}{2\left(\omega_{1}^{2}-\omega^{2}\right)}-\frac{F_{1}^{2}}{4 \omega^{2} a_{1}^{2}}\right]=0 \\
\sigma_{2}^{2} & -\frac{\gamma \omega_{1}}{\omega_{1}^{2}-\omega^{2}} \sigma_{2}+\left(\frac{\omega_{1}^{2} \gamma^{2}}{4\left(\omega_{1}^{2}-\omega^{2}\right)^{2}}+\zeta_{2}^{2} \omega_{1}^{2}+\frac{\omega_{1}^{4} \zeta_{1}^{2}}{4\left(\omega_{1}^{2}-\omega^{2}\right)^{2}}\right. \\
& \left.+\frac{\omega_{1}^{3} \zeta_{1} \zeta_{2}}{\omega_{1}^{2}-\omega^{2}}-\frac{F_{2}^{2} \omega_{1}^{2}}{4 a_{2}^{2}\left(\omega^{2}-\Omega_{2}^{2}\right)^{2}}\right)=0
\end{aligned}
$$

and case (3) is represented by equations (37) and (38).

\subsection{Results and discussion}

Fig. 5 shows the effects of the detuning parameter $\sigma_{1}$ on the steady state amplitude of the main system $a_{1}$ for the stability first case where $a_{1} \neq 0$ and $a_{2}=0$. Fig. 5 (a) shows that the curves converges or diverges as $\gamma$ is decreasing or increasing, leading to the appearance of the jump phenomenon. Figs. 5 ((b) and

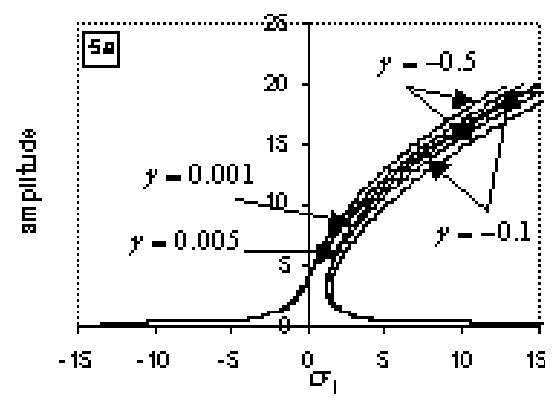

(a) Effects of non-linear parameter $\gamma$

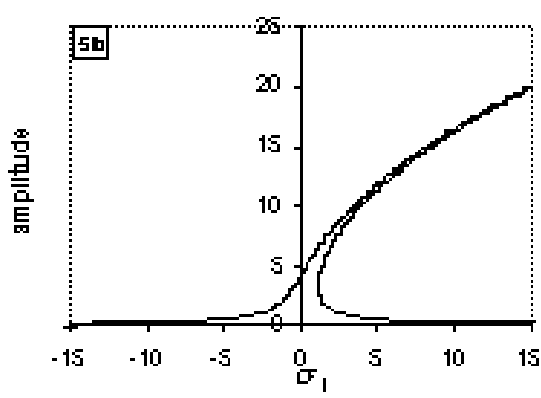

(b) Effects of natural frequency $\omega_{1}$

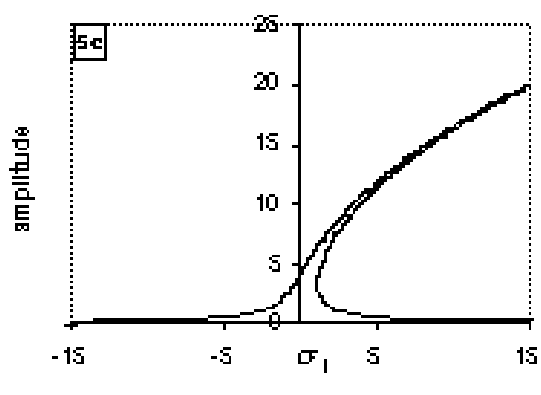

(c) Effects of damping coefficient $\zeta_{1}$

Fig. 5 . 
(c)) show that the curves are bent to the right leading to the appearance of the jump phenomenon and the effect of increasing or decreasing $\omega_{1}$ and $\zeta_{1}$ trivial.

Fig. 6 shows the effects of the detuning parameter $\sigma_{2}$ on the steady state amplitude of the main system $a_{2}$ for the stability second case $a_{2} \neq 0$ and $a_{1}=0$, not leading to the appearance of the jump phenomenon. Fig. 6 (a) shows that the positive and negative values of $\gamma$, the curve is neither bent to the right nor the left. Figs. $6((\mathrm{~b})$ and $(\mathrm{g}))$ indicate that the steady state amplitude is monotonic decreasing function in natural frequency $\omega$ and in the excitation frequency $\Omega_{2}$. Figs. 6 ((c)

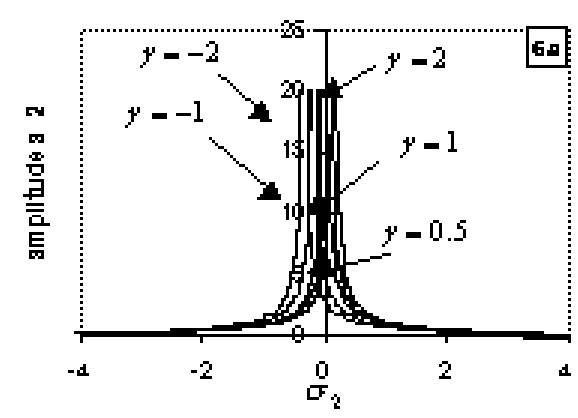

(a) Effects of non-linear parameter $\gamma$

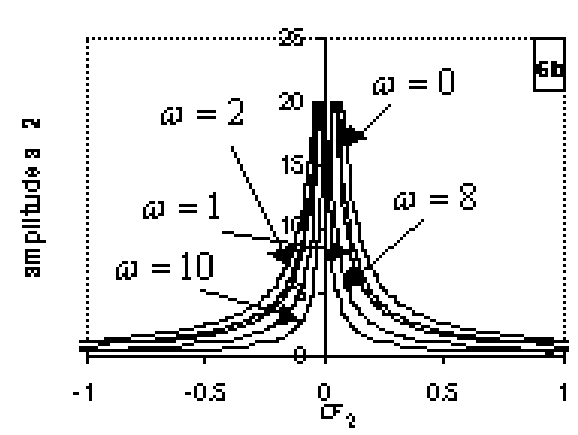

(b) Effects of natural frequency $\omega$

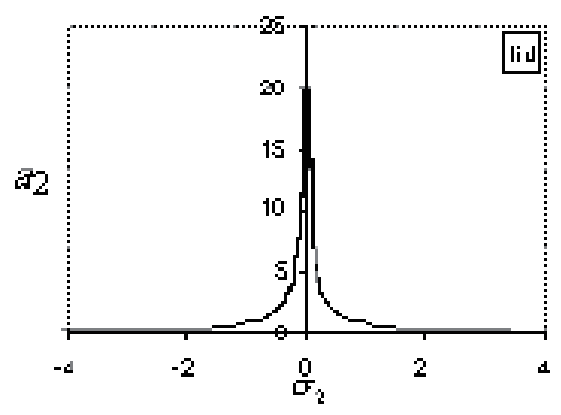

(d) Effects of damping coefficient $\zeta_{1}$

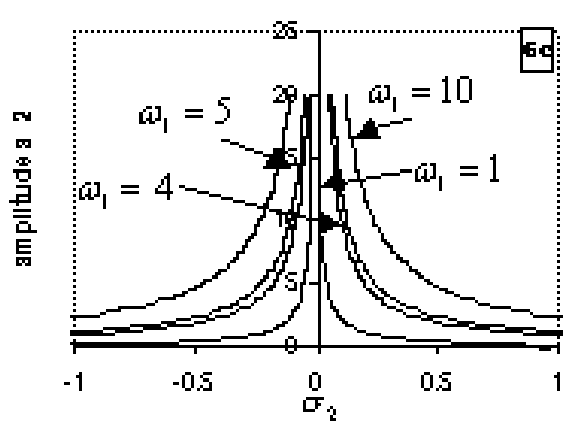

(c) Effects of natural frequency $\omega_{1}$

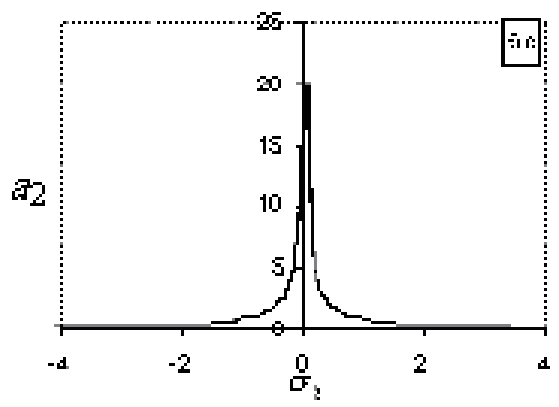

(e) Effects of damping coefficient $\zeta_{2}$ 


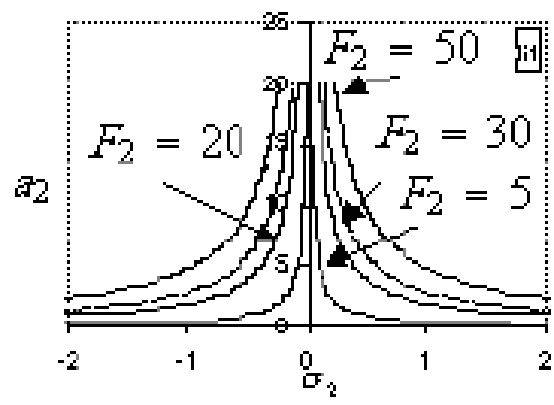

(f) Effects of excitation amplitude $F_{2}$

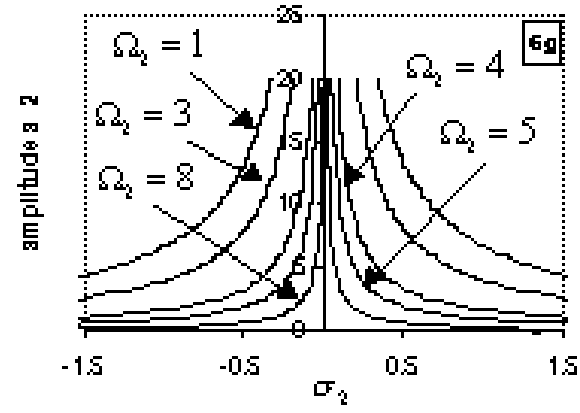

(g) Effects of excitation frequency $\Omega_{2}$

Fig. 6 .

and (f)) show that the steady state amplitude $a_{2}$ is a monotonic decreasing function in the natural frequency $\omega_{1}$ and increasing function in the excitation amplitude $F_{2}$. Figs. $6((\mathrm{~d})$ and $(\mathrm{e}))$ show increasing or decreasing $\zeta_{1}$ and $\zeta_{2}$ do not effect the steady state amplitude $a_{2}$.

Fig. 7 shows that the steady state amplitude without absorber at primary resonance where, $\Omega_{1}=\omega$ is about $100 \%$ of the maximum excitation amplitude $F_{1}$, the system is stable and free of dynamic chaos.

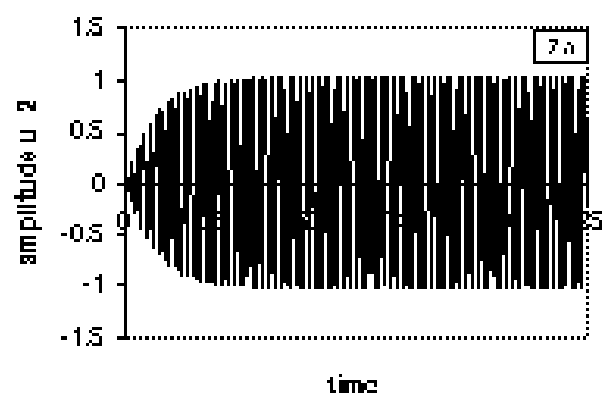

Fig. 7. System behavior at primary resonance.

\section{Effects of the TMA}

Fig. 8 illustrates the response for the system with absorber at the simultaneous primary resonance $\Omega_{1} \cong \omega \cong \omega_{1}$. The effectiveness of the absorber $E_{a}$ (steady state of the wing without absorber /steady state with absorber) is increased about 125 . The phase plane is approximately limit cycle. 

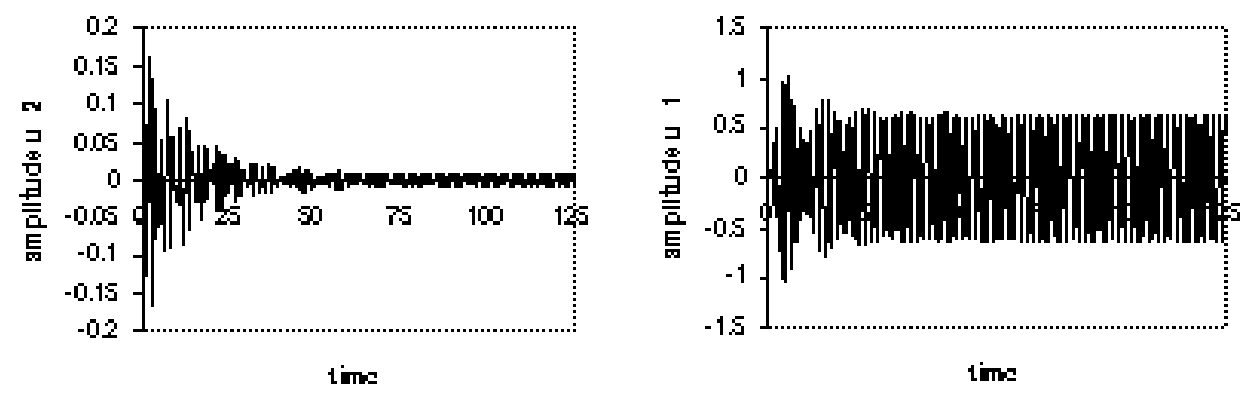

Fig. 8. System behavior with absorber at the simultaneous primary resonance $\Omega_{1} \cong \omega \cong \omega_{1} . \omega=\omega_{1}=\Omega_{1}=4, \alpha=0.1, \beta=0.1, \gamma=1.6, \zeta=0.03$, $\zeta_{1}=0.005, \zeta_{2}=0.00625, F_{1}=1, F_{2}=0.0025, \Omega_{2}=7.5$.

\section{Active Control}

Here, active control is applied to improve the behavior of the system. A second order non-linear controller is added to the main system which represents a 1:2 internal resonance active control absorber of a vibrating aircraft wing in the following form [20], [21] and [22].

$$
\begin{aligned}
& \ddot{u}_{1}+2 \varepsilon \zeta_{1} \omega_{1} \dot{u}_{1}+\omega_{1}^{2} u_{1}=\varepsilon g_{12} u_{1} u_{2} \\
& \ddot{u}_{2}+2 \varepsilon \zeta_{2} \omega_{2} \dot{u}_{2}+\omega_{2}^{2} u_{2}+\varepsilon \alpha u_{2}^{2}+\varepsilon \beta u_{2}^{3}=\varepsilon g_{11} u_{1}^{2}+\varepsilon \sum_{j=1}^{2} F_{j} \cos \left(\Omega_{j} T_{0}\right)
\end{aligned}
$$

where $u_{1}$ denotes the response of a second-order controller, $\omega_{1}$ is the natural frequency of the controller, $\zeta_{1}$ is the damping ratio of the controller, $u_{2}$ represents one of the modal co-ordinates of the wing, $\omega_{2}$ is this modal frequency, $\zeta_{2}$ is the damping ratio, $g_{11}$ and $g_{12}$ are positive gain constants, $F$ is the amplitude of the external excitation force, $\Omega_{j}$ are the external excitation frequencies, $\omega_{2}$ is close to $2 \omega_{1}$ (i.e. 1:2), $\Omega_{j}$ is close to $\omega_{2}, t$ is the time, $\alpha$ and $\beta$ are coefficients of non-linear terms, $\varepsilon$ is a small perturbation parameter. The analytic solutions of equations (39) and (40) are obtained assuming that the coefficients of $\zeta_{1} \omega_{1}, \zeta_{2} \omega_{2}, g_{12}, g_{11}, F, \alpha$ and $\beta$ are small. Following the same procedure, we get:

$$
\begin{aligned}
u_{10}\left(T_{0}, T_{n}\right)= & A_{0}\left(T_{n}\right) e^{i \omega_{1} T_{0}}+c c \\
u_{20}\left(T_{0}, T_{n}\right)= & B_{0}\left(T_{n}\right) e^{i \omega_{2} T_{0}}+c c \\
u_{11}\left(T_{0}, T_{n}\right)= & C_{1} e^{i \omega_{1} T_{0}}+C_{2} e^{i\left(\omega_{1}+\omega_{2}\right) T_{0}}+C_{3} e^{i\left(\omega_{1}-\omega_{2}\right) T_{0}}+c c \\
u_{21}\left(T_{0}, T_{n}\right)= & C_{4} e^{i \omega_{2} T_{0}}+C_{5} e^{2 i \omega_{2} T_{0}}+C_{6} e^{3 i \omega_{2} T_{0}}+C_{7} e^{i \Omega_{1} T_{0}}+C_{8} e^{i \Omega_{2} T_{0}} \\
& +C_{9}+c c \\
u_{12}\left(T_{0}, T_{n}\right)= & F_{1} e^{i \omega_{1} T_{0}}+F_{2} e^{i\left(\omega_{1}+\omega_{2}\right) T_{0}}+F_{3} e^{i\left(\omega_{1}-\omega_{2}\right) T_{0}}+F_{4} e^{i\left(\omega_{1}+2 \omega_{2}\right) T_{0}}
\end{aligned}
$$




$$
\begin{aligned}
& +F_{5} e^{i\left(\omega_{1}-2 \omega_{2}\right) T_{0}}+F_{6} e^{i\left(\omega_{1}+3 \omega_{2}\right) T_{0}}+F_{7} e^{i\left(\omega_{1}-3 \omega_{2}\right) T_{0}}+F_{8} e^{i\left(\omega_{1}+\Omega_{1}\right) T_{0}} \\
& +F_{9} e^{i\left(\omega_{1}-\Omega_{1}\right) T_{0}}+F_{10} e^{i\left(\omega_{1}+\Omega_{2}\right) T_{0}}+F_{11} e^{i\left(\omega_{1}-\Omega_{2}\right) T_{0}}+c c \\
& u_{22}\left(T_{0}, T_{n}\right)=g_{1} e^{i \omega_{2} T_{0}}+g_{2} e^{2 i \omega_{2} T_{0}}+g_{3} e^{3 i \omega_{2} T_{0}}+g_{4} e^{4 i \omega_{2} T_{0}}+g_{5} e^{5 i \omega_{2} T_{0}}+g_{6} e^{2 i \omega_{1} T_{0}} \\
& +g_{7} e^{i\left(\omega_{2}+2 \omega_{1}\right) T_{0}}+g_{8} e^{i\left(\omega_{2}-2 \omega_{1}\right) T_{0}}+g_{9} e^{i \Omega_{1} T_{0}}+g_{10} e^{i \Omega_{2} T_{0}} \\
& +g_{11} e^{i\left(\omega_{2}+\Omega_{1}\right) T_{0}}+g_{12} e^{i\left(\omega_{2}-\Omega_{1}\right) T_{0}}+g_{13} e^{i\left(\omega_{2}+\Omega_{2}\right) T_{0}}+g_{14} e^{i\left(\omega_{2}-\Omega_{2}\right) T_{0}} \\
& +g_{15} e^{i\left(2 \omega_{2}+\Omega_{1}\right) T_{0}}+g_{16} e^{i\left(2 \omega_{2}-\Omega_{1}\right) T_{0}}+g_{17} e^{i\left(2 \omega_{2}+\Omega_{2}\right) T_{0}} \\
& +g_{18} e^{i\left(2 \omega_{2}-\Omega_{2}\right) T_{0}}+g_{19}+c c \\
& u_{13}\left(T_{0}, T_{n}\right)=h_{1} e^{i \omega_{1} T_{0}}+h_{2} e^{3 i \omega_{1} T_{0}}+h_{3} e^{i\left(\omega_{1}+\omega_{2}\right) T_{0}}+h_{4} e^{i\left(\omega_{1}-\omega_{2}\right) T_{0}}+h_{5} e^{i\left(\omega_{1}+2 \omega_{2}\right) T_{0}} \\
& +h_{6} e^{i\left(\omega_{1}-2 \omega_{2}\right) T_{0}}+h_{7} e^{i\left(\omega_{1}+3 \omega_{2}\right) T_{0}}+h_{8} e^{i\left(\omega_{1}-3 \omega_{2}\right) T_{0}}+h_{9} e^{i\left(\omega_{1}+4 \omega_{2}\right) T_{0}} \\
& +h_{10} e^{i\left(\omega_{1}-4 \omega_{2}\right) T_{0}}+h_{11} e^{i\left(\omega_{1}+5 \omega_{2}\right) T_{0}}+h_{12} e^{i\left(\omega_{1}-5 \omega_{2}\right) T_{0}} \\
& +h_{13} e^{i\left(\omega_{1}+\Omega_{1}\right) T_{0}}+h_{14} e^{i\left(\omega_{1}-\Omega_{1}\right) T_{0}}+h_{15} e^{i\left(\omega_{1}+\Omega_{2}\right) T_{0}}+h_{16} e^{i\left(\omega_{1}-\Omega_{2}\right) T_{0}} \\
& +h_{17} e^{i\left(\Omega_{1}+\left(\omega_{1}+\omega_{2}\right)\right) T_{0}}+h_{18} e^{i\left(\Omega_{1}-\left(\omega_{1}+\omega_{2}\right)\right) T_{0}}+h_{19} e^{i\left(\Omega_{2}+\left(\omega_{1}+\omega_{2}\right)\right) T_{0}} \\
& +h_{20} e^{i\left(\Omega_{2}-\left(\omega_{1}+\omega_{2}\right)\right) T_{0}}+h_{21} e^{i\left(\Omega_{1}+\left(\omega_{1}-\omega_{2}\right)\right) T_{0}}+h_{22} e^{i\left(\Omega_{1}-\left(\omega_{1}-\omega_{2}\right)\right) T_{0}} \\
& +h_{23} e^{i\left(\Omega_{2}+\left(\omega_{1}-\omega_{2}\right)\right) T_{0}}+h_{24} e^{i\left(\Omega_{2}-\left(\omega_{1}-\omega_{2}\right)\right) T_{0}}+h_{25} e^{i\left(\Omega_{1}+\left(\omega_{1}+2 \omega_{2}\right)\right) T_{0}} \\
& +h_{26} e^{i\left(\Omega_{1}-\left(\omega_{1}+2 \omega_{2}\right)\right) T_{0}}+h_{27} e^{i\left(\Omega_{2}+\left(\omega_{1}+2 \omega_{2}\right)\right) T_{0}}+h_{28} e^{i\left(\Omega_{2}-\left(\omega_{1}+2 \omega_{2}\right)\right) T_{0}} \\
& +h_{29} e^{i\left(\Omega_{1}+\left(\omega_{1}-2 \omega_{2}\right)\right) T_{0}}+h_{30} e^{i\left(\Omega_{1}-\left(\omega_{1}-2 \omega_{2}\right)\right) T_{0}}+h_{31} e^{i\left(\Omega_{2}+\left(\omega_{1}-2 \omega_{2}\right)\right) T_{0}} \\
& +h_{32} e^{i\left(\Omega_{2}-\left(\omega_{1}-2 \omega_{2}\right)\right) T_{0}}+h_{33} e^{i\left(3 \omega_{1}+\omega_{2}\right) T_{0}}+h_{34} e^{i\left(3 \omega_{1}-\omega_{2}\right) T_{0}}+c c
\end{aligned}
$$

$$
\begin{aligned}
& u_{23}\left(T_{0}, T_{n}\right)=k_{1} e^{i \omega_{2} T_{0}}+k_{2} e^{2 i \omega_{2} T_{0}}+k_{3} e^{3 i \omega_{2} T_{0}}+k_{4} e^{4 i \omega_{2} T_{0}}+k_{5} e^{5 i \omega_{2} T_{0}}+k_{6} e^{6 i \omega_{2} T_{0}} \\
& +k_{7} e^{7 i \omega_{2} T_{0}}+k_{8} e^{i \omega_{1} T_{0}}+k_{9} e^{2 i \omega_{1} T_{0}}+k_{10} e^{3 i \omega_{1} T_{0}}+k_{11} e^{4 i \omega_{1} T_{0}} \\
& +k_{12} e^{i\left(2 \omega_{1}+\omega_{2}\right) T_{0}}+k_{13} e^{i\left(2 \omega_{1}-\omega_{2}\right) T_{0}}+k_{14} e^{i\left(2 \omega_{1}+2 \omega_{2}\right) T_{0}} \\
& +k_{15} e^{i\left(2 \omega_{1}-2 \omega_{2}\right) T_{0}}+k_{16} e^{i\left(2 \omega_{1}+3 \omega_{2}\right) T_{0}}+k_{17} e^{i\left(2 \omega_{1}-3 \omega_{2}\right) T_{0}} \\
& +k_{18} e^{i\left(2 \omega_{1}+4 \omega_{2}\right) T_{0}}+k_{19} e^{i\left(2 \omega_{1}-4 \omega_{2}\right) T_{0}}+k_{20} e^{i \Omega_{1} T_{0}}+k_{21} e^{i \Omega_{2} T_{0}} \\
& +k_{22} e^{2 i \Omega_{1} T_{0}}+k_{23} e^{2 i \Omega_{2} T_{0}}+k_{24} e^{i\left(\Omega_{1}+\Omega_{2}\right) T_{0}}+k_{25} e^{i\left(\Omega_{2}-\Omega_{1}\right) T_{0}} \\
& +k_{26} e^{i\left(\Omega_{1}+2 \omega_{1}\right) T_{0}}+k_{27} e^{i\left(\Omega_{1}-2 \omega_{1}\right) T_{0}}+k_{28} e^{i\left(\Omega_{2}+2 \omega_{1}\right) T_{0}} \\
& +k_{29} e^{i\left(\Omega_{2}-2 \omega_{1}\right) T_{0}}+k_{30} e^{i\left(\Omega_{1}+\omega_{2}\right) T_{0}}+k_{31} e^{i\left(\Omega_{1}-\omega_{2}\right) T_{0}} \\
& +k_{32} e^{i\left(\Omega_{2}+\omega_{2}\right) T_{0}}+k_{33} e^{i\left(\Omega_{2}-\omega_{2}\right) T_{0}}+k_{34} e^{i\left(\Omega_{1}+2 \omega_{2}\right) T_{0}} \\
& +k_{35} e^{i\left(\Omega_{1}-2 \omega_{2}\right) T_{0}}+k_{36} e^{i\left(\Omega_{2}+2 \omega_{2}\right) T_{0}}+k_{37} e^{i\left(\Omega_{2}-2 \omega_{2}\right) T_{0}} \\
& +k_{38} e^{i\left(\Omega_{1}+3 \omega_{2}\right) T_{0}}+k_{39} e^{i\left(\Omega_{1}-3 \omega_{2}\right) T_{0}}+k_{40} e^{i\left(\Omega_{2}+3 \omega_{2}\right) T_{0}} \\
& +k_{41} e^{i\left(\Omega_{2}-3 \omega_{2}\right) T_{0}}+k_{42} e^{i\left(\Omega_{1}+4 \omega_{2}\right) T_{0}}+k_{43} e^{i\left(\Omega_{1}-4 \omega_{2}\right) T_{0}} \\
& +k_{44} e^{i\left(\Omega_{2}+4 \omega_{2}\right) T_{0}}+k_{45} e^{i\left(\Omega_{2}-4 \omega_{2}\right) T_{0}}+k_{46} e^{i\left(2 \Omega_{1}+\omega_{2}\right) T_{0}} \\
& +k_{47} e^{i\left(2 \Omega_{1}-\omega_{2}\right) T_{0}}+k_{48} e^{i\left(2 \Omega_{2}+\omega_{2}\right) T_{0}}+k_{49} e^{i\left(2 \Omega_{2}-\omega_{2}\right) T_{0}} \\
& +k_{50} e^{i\left(\omega_{2}+\left(\Omega_{1}+\Omega_{2}\right)\right) T_{0}}+k_{51} e^{i\left(\omega_{2}-\left(\Omega_{1}+\Omega_{2}\right)\right) T_{0}}+k_{52} e^{i\left(\omega_{2}+\left(\Omega_{1}-\Omega_{2}\right)\right) T_{0}} \\
& +k_{53} e^{i\left(\omega_{2}-\left(\Omega_{1}-\Omega_{2}\right)\right) T_{0}}+k_{54} e^{i\left(\Omega_{1}+\left(2 \omega_{1}+\omega_{2}\right)\right) T_{0}}+k_{55} e^{i\left(\Omega_{1}-\left(2 \omega_{1}+\omega_{2}\right)\right) T_{0}} \\
& +k_{56} e^{i\left(\Omega_{2}+\left(2 \omega_{1}+\omega_{2}\right)\right) T_{0}}+k_{57} e^{i\left(\Omega_{2}-\left(2 \omega_{1}+\omega_{2}\right)\right) T_{0}}+k_{58} e^{i\left(\Omega_{1}+\left(2 \omega_{1}-\omega_{2}\right)\right) T_{0}}
\end{aligned}
$$




$$
\begin{aligned}
& +k_{59} e^{i\left(\Omega_{1}-\left(2 \omega_{1}-\omega_{2}\right)\right) T_{0}}+k_{60} e^{i\left(\Omega_{2}+\left(2 \omega_{1}-\omega_{2}\right)\right) T_{0}}+k_{61} e^{i\left(\Omega_{2}-\left(2 \omega_{1}-\omega_{2}\right)\right) T_{0}} \\
& +k_{62} e^{i\left(4 \omega_{1}+\omega_{2}\right) T_{0}}+k_{63} e^{i\left(4 \omega_{1}-\omega_{2}\right) T_{0}}+k_{64}+c c
\end{aligned}
$$

where $A_{0}, B_{0}, C_{i}, F_{m}, g_{n}, h_{s}, k_{l}:(i=1,2, \ldots, 9),(m=1,2, \ldots, 11),(n=$ $1,2, \ldots, 19),(s=1,2, \ldots, 34)$ and $(l=1,2, \ldots, 64)$ are complex functions in $T_{n}$. From the above analysis the general solutions of $u_{1}$ and $u_{2}$ are:

$$
\begin{aligned}
& u_{1}=u_{10}+\varepsilon u_{11}+\varepsilon^{2} u_{12}+\varepsilon^{3} u_{13}+O\left(\varepsilon^{4}\right) \\
& u_{2}=u_{20}+\varepsilon u_{21}+\varepsilon^{2} u_{22}+\varepsilon^{3} u_{23}+O\left(\varepsilon^{4}\right)
\end{aligned}
$$

From the above solutions many resonance cases are shown in Table 4.

Table 4 .

\begin{tabular}{|c|l|l|}
\hline No. & Type of Resonances & \multicolumn{1}{c|}{ Case } \\
\hline 1 & trivial resonance & $\Omega_{1} \cong \Omega_{2} \cong \omega_{1} \cong \omega_{2}=0$ \\
\hline 2 & primary resonance & $\omega_{2} \cong \omega_{1}, \omega_{2} \cong \Omega_{1}, \omega_{2} \cong \Omega_{2}$ \\
\hline 3 & sub-harmonic resonances & $\Omega_{1} \cong n \omega_{2}, \Omega_{2} \cong n \omega_{2}, \omega_{2} \cong m \omega_{1}, \Omega_{1} \cong 2 \omega_{1}$, \\
& & $n=2,3,4,5$ and $m=2,3,4$ \\
\hline 4 & super-harmonic resonances & $\omega_{2}=\omega_{1} / 2, \omega_{2}=2 \omega_{1} / 3, \omega_{2}=2 \omega_{1} / 5$, \\
& & $\Omega_{1} \cong \omega_{2} / 2, \Omega_{2} \cong \omega_{2} / 2$ \\
\hline 5 & combination resonances & $\pm \omega_{2} \cong \pm \Omega_{1} \pm \Omega_{2}, \pm \omega_{2} \cong\left( \pm \Omega_{1} \pm \Omega_{2}\right) / 2$, \\
& & $\pm \Omega_{1} \cong \pm \omega_{2} \pm 2 \omega_{1}, \pm \Omega_{2} \cong \pm \omega_{2} \pm 2 \omega_{1}$, \\
& & $\pm \Omega_{1} \cong \pm 2\left(\omega_{2} \pm \omega_{1}\right), \pm \Omega_{2} \cong 2\left( \pm \omega_{2} \pm \omega_{1}\right)$ \\
\hline 6 & simultaneous resonance & any combination of the two resonance cases \\
& & are classified as simultaneous resonance \\
\hline
\end{tabular}

Following the same procedure as in part 2, we get

$$
\sigma_{1}=-\frac{3 \beta a_{2}^{2}}{8 \omega_{2}} \pm \sqrt{F_{1}^{2}-4 a_{2}^{2} \zeta_{2}^{2} \omega_{2}^{4}}
$$

Fig. $9(\mathrm{a})-(\mathrm{d})$ show the effects of the non-linear parameter $\beta$, the natural frequency $\omega_{2}$, the damping coefficient $\zeta_{2}$, and the excitation amplitudes $F_{1}$ on the steady state amplitude " $a_{2}$ ". Fig. 9 (a) shows that the positive and negative values of $\beta$, produce either hard or soft spring respectively as the curve is either bent to the left or the right, leading to the appearance of the jump phenomenon. Fig. 9 (b) indicates that the steady state amplitude is a monotonic increasing function in $\omega_{2}$ also for the decreasing natural frequency $\omega_{2}$, the curve is bent to the left leading to the occurrence of the jump phenomena. Fig. 9 (c) shows that the curve is bent to the left, leading to the occurrence of the jump phenomena and the effect of increasing or decreasing $\zeta_{2}$ is insignificant indicating the occurrence of saturation phenomena. Fig. 9 (d) shows that the steady state amplitude is a monotonic increasing function in the excitation amplitude $F_{1}$. 


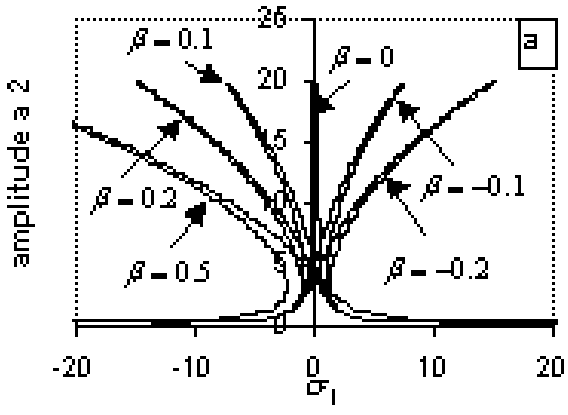

(a) Effects of non-linear parameter $\beta$

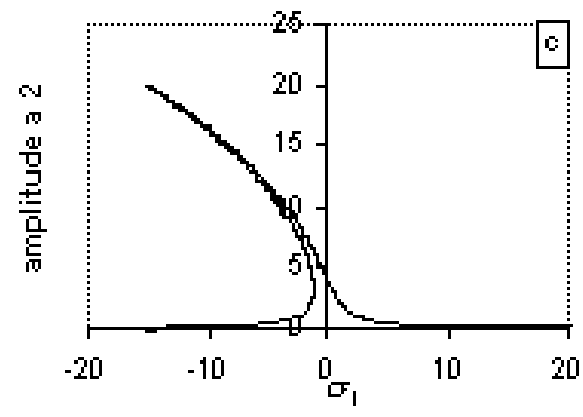

(c) Effects of damping coefficient $\zeta$

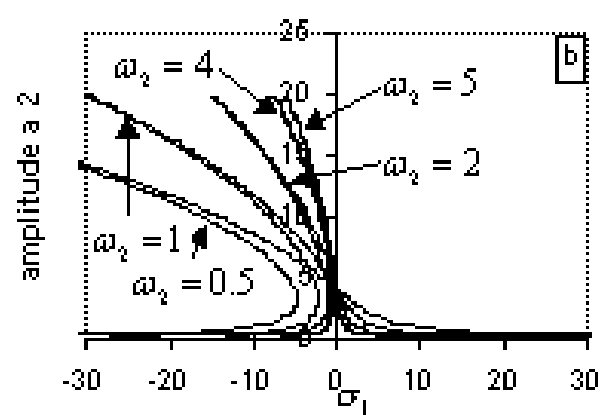

(b) Effects of natural frequency $\omega_{2}$

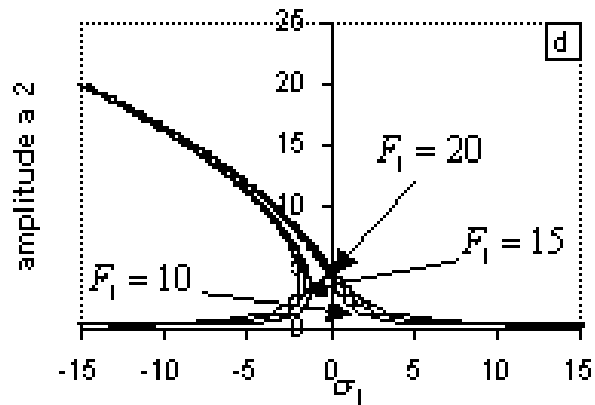

(d) Effects of excitation amplitude $F_{1}$

Fig. 9.

Fig. 10 illustrates the response for the system with active controller at primary resonance where $\Omega_{1} \cong \omega_{2}$ and internal resonance where $\omega_{2}=2 \omega_{1}$. The effectiveness of the absorber $E_{a}$ is about 8 .

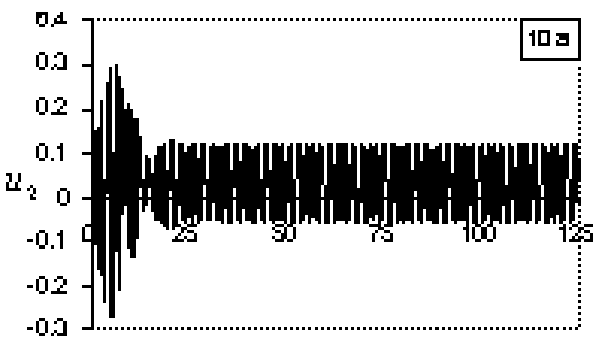

$\operatorname{tims}$

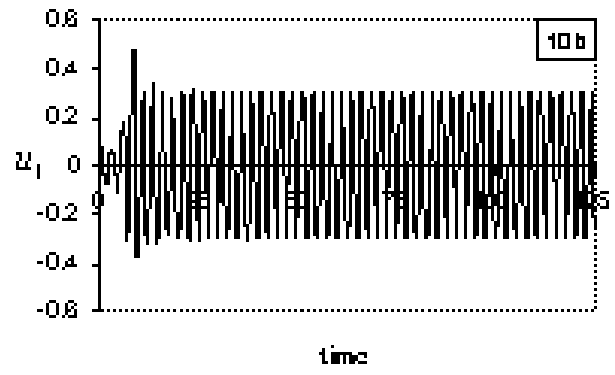

Fig. 10. System behavior with active controller at primary resonance where $\Omega_{1} \cong \omega_{2}$ and internal resonance where $\omega_{2}=2 \omega_{1} . \zeta_{2}=0.05, \omega_{2}=5, \alpha=0.1, \beta=0.2$, $\zeta_{1}=0.05, F_{1}=1, F_{2}=0.005, \Omega_{1}=5, \Omega_{2}=8, \omega_{1}=2.5, g_{12}=20, g_{11}=18$. 


\section{Conclusions}

The main system of non-linear differential equation representing the vibration of an aircraft wing subjected to multi-excitation force is considered and solved using the method of multiple scale perturbation. Two main strategies are used. They are passive and active control methods. A tuned mass absorber is applied to the system at simultaneous primary resonance (passive control) and the same system is considered with 1:2 internal resonance controller (active control). The analytical solution is derived up to the fourth order approximation and its stability is obtained and studied applying frequency response functions. From the above study the following conclusion can be deduced.

(i) For the main system:

1. The steady state amplitudes is monotonic increasing function to the maximum excitation amplitude $F_{1}$.

2. The effects on steady state amplitudes is insignificant for increasing or decreasing values of $\zeta$. The curve is bent to the right, leading to the occurrence of the jump phenomena.

3. The positive and negative values of $\beta$, produce either hard or soft spring respectively as the curve is either bent to the right or the left, leading to the appearance of the jump phenomenon.

4. For the small values of natural frequency the curve is bent to the right leading to the appearance of the jump phenomenon.

(ii) For passive control

1. The steady state amplitudes is monotonic increasing functions to the maximum excitation amplitude $F_{1}$.

2. The steady state amplitude insignificant for increasing or decreasing values of $\zeta_{1}$. The curve is bent to the right, leading to the occurrence of the jump phenomena.

3. The positive and negative values of $\beta$, produce either hard or soft spring respectively as the curve is either bent to the right or the left, leading to the appearance of the jump phenomenon.

4. For the small values of natural frequency the curve is bent to the right leading to the appearance of the jump phenomenon.

5. The effectiveness of the absorber is about $E_{a}=125$ at primary resonance where $\Omega_{1}=\omega$ and internal resonance $\omega=\omega_{1}$.

(iii) For active control

1. The steady state amplitudes is monotonic increasing function to the maximum excitation amplitude $F_{1}$.

2. The steady state amplitudes is insignificant for increasing or decreasing value of $\zeta_{2}$. The curve is bent to the left, leading to the occurrence of the jump phenomena. 
3. The positive and negative values of $\beta$, produce either hard or soft spring respectively as the curve is either bent to the left or the right, leading to the appearance of the jump phenomenon and vice versa at active control.

4. For the small values of natural frequency the curve is bent to the left leading to the appearance of the jump phenomenon.

5. The effectiveness of the absorber is reduce to about $E_{a}=8$ at primary resonance $\Omega_{1}=\omega_{2}$ and internal resonance $\omega_{2}=2 \omega_{1}$.

It is worth to mention that passive control if possible has the priority over the active control from both the economical and stability problems point if view.

\section{References}

[1] J.D. Mead, Passive Vibration Control. John Wiley \& Sons, 1999.

[2] M. Eissa, Vibration and chaos control in I.C engines subject to harmonic torque via nonlinear absorbers. ISMV-2000, Second International Symposium on Mechanical Vibrations, Islamabad, Pakistan, 2000.

[ 3 ] M. Eissa, Vibration control of non-linear mechanical system via a neutralizer. Electronic Bulletin, No. 16, Faculty of Electronic Engineering, Menouf, Egypt, 1999, July.

[4] Cheng-Tang Lee et al., Sub-harmonic vibration absorber for rotating machinery. ASME Journal of Vibration and Acoustics, 119 (1997), 590-595.

[ 5 ] M. Eissa and El-Ganaini, Multi absorbers for vibration control of non-linear structures to harmonic excitations, Part I. ISMV Conference, Islamabad, Pakistan, 2000.

[6] M. Eissa and El-Ganaini, Multi absorbers for vibration control of non-linear structures to harmonic excitations, Part II. ISMV Conference, Islamabad, Pakistan, 2000.

[ 7 ] I.Y. Shen, W. Guo and Y.C. Pao, Torsional vibration control of a shaft through active constrained layer damping treatments. Journal of Vibration and Acoustics, 119 (1997), $504-511$.

[ 8 ] N. Liu and K.W. Wang, A non-dimensional parametric study of enhanced active constrained layer damping treatments. Journal of Sound and Vibration, 223, No.4 (1999), 611-644.

[ 9 ] R. Stanawy and D. Chantalkhana, Active constrained layer damping of clamped-clamped plate vibration. Journal of Sound and Vibration, 241, No.5 (2001), 755-777.

[10] A. Baz, M.C. Ray and J. Oh, Active constrained layer damping of thin cylindrical shell. Journal of Sound and Vibration, 240, No.5 (2001), 921-935.

[11] Y.M. Shi, Z.F. Li, X.H. Hua, Z.F. Fu and T.X. Liu, The modeling and vibration control of beams with active constrained layer. Journal of Sound and Vibration, 245, No.5 (2001), $785-800$.

[12] D. Sun and L. Tong, Modeling and vibration control of beams with partially de-bonded active constrained layer damping patch. Journal of Sound and Vibration, 252, No.3 (2002), 493-507.

[13] A.H. Nayfeh and D.T. Mook, Non-linear Oscillations. New York, Wiley, 1979.

[14] P.F. Pai and A.H. Nayfeh, Three-dimensional non-linear vibrations of composite beams-II. Flapwise excitations. Non-linear Dynamics, 2 (1991), 1-34.

[15] M.F. Golnaraghi, Regulation of flexible structures via non-linear coupling. Journal of Dynamics and Control, 1 (1991), 405-428.

[16] S.S. Oueini and A.H. Nayfeh, Saturation control of a DC motor. AIAA-96-1642-CP, 1996.

[17] S.S. Oueini, A.H. Nayfeh and M.F. Golnaraghi, A theoretical and experimental implementation of a control method based on saturation. Non-linear Dynamics, 13 (1997), 189-202.

[18] S.S. Oueini and M.F. Golnaraghi, Experimental implementation of the internal resonance control strategy. Journal of Sound and Vibration, 191, No.3 (1996), 369-377.

[19] C. Park, C. Walz and I. Chopra, Bending and torsion models of beams with induced strain actuators. The SPIE Smart Structures and Materials 93, Alburquerque, NM, 1993.

[20] B. Wen, A.S. Naser and M.J. Schulz, Structural vibration control using PZT patches and non-linear phenomena. Journal of Sound and Vibration, 215, No.2 (1998), 273-296. 
[21] P.F. Pai and M.J. Schulz, A refined non-linear vibration absorber. International Journal of Mechanical Sciences, 42 (2000), 537-560.

[22] P.F. Pai, B. Rommel and M.J. Schulz, Non-linear vibration absorbers using higher order internal resonances. Journal of Sound and vibration, 234, No.5 (2000), 799-817. 